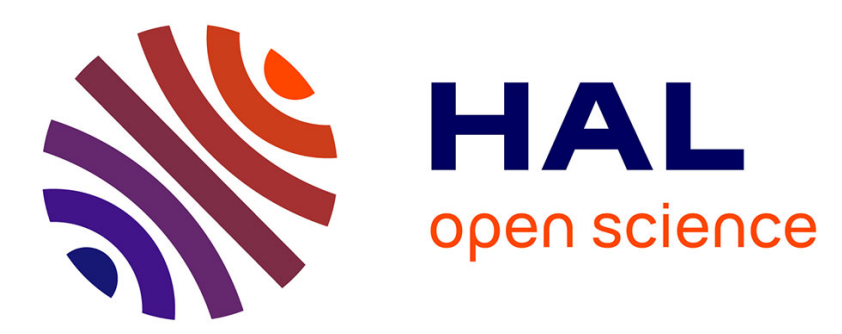

\title{
Multi-scale modeling of diffusion and electrochemical reactions in porous micro-electrodes
}

T.D. D Le, Didier Lasseux, X.P. P Nguyen, G. Vignoles, N. Mano, A. Kuhn

\section{To cite this version:}

T.D. D Le, Didier Lasseux, X.P. P Nguyen, G. Vignoles, N. Mano, et al.. Multi-scale modeling of diffusion and electrochemical reactions in porous micro-electrodes. Chemical Engineering Science, 2017, 173, pp.153-167. 10.1016/j.ces.2017.07.039 . hal-03140930

\author{
HAL Id: hal-03140930 \\ https://hal.science/hal-03140930
}

Submitted on 14 Feb 2021

HAL is a multi-disciplinary open access archive for the deposit and dissemination of scientific research documents, whether they are published or not. The documents may come from teaching and research institutions in France or abroad, or from public or private research centers.
L'archive ouverte pluridisciplinaire HAL, est destinée au dépôt et à la diffusion de documents scientifiques de niveau recherche, publiés ou non, émanant des établissements d'enseignement et de recherche français ou étrangers, des laboratoires publics ou privés. 


\title{
Multi-scale modeling of diffusion and electrochemical reactions in porous micro-electrodes
}

\author{
T.D. Le ${ }^{a}$, D. Lasseux ${ }^{a, *}$, X.P. Nguyen ${ }^{a}$, G. Vignoles ${ }^{b}$, N. Mano ${ }^{c}$, A. Kuhn ${ }^{d}$ \\ a Institut de Mécanique et d'Ingénierie, CNRS UMR5295, Esplanade des Arts et Métiers, Talence Cedex 33405, France \\ b Laboratoire des Composites ThermoStructuraux, CNRS UMR5801, 3 Allée de la Boétie, 33600 Pessac, France \\ 'Centre de Recherche Paul Pascal, CNRS UPR8641, University of Bordeaux, Avenue Albert Schweitzer, 33600 Pessac, France \\ ${ }^{\mathrm{d}}$ Institut des Sciences Moléculaires, CNRS UMR5255, University of Bordeaux ENSCBP, 16 Avenue Pey Berland, 33607 Pessac, France
}

\section{H I G H L I G H T S}

- A diffusion and electrochemical reaction model in a porous electrode is proposed.

- A macroscale model operating at the electrode scale is obtained by upscaling.

- The macroscale model is validated by direct numerical simulation and experiments.

Keywords:

Porous electrode

Diffusion

Heterogeneous reaction

Volume averaging method

\begin{abstract}
A B S T R A C T
A multi-scale model of diffusion/reaction at play in a porous electrode is developed and solutions to the physico-electro-chemical coupled problem are provided. This represents a key step to progress in the optimization of new efficient and innovative micro-electro-devices that needs to be addressed from a chemical engineering point of view. The pore-scale model based on Fickian diffusion in the porous medium and Nernstian layer and the electrochemical reaction governed by the Buttler-Volmer equation is upscaled using volume averaging to obtain a macroscopic model that describes the process on an effective equivalent medium. The validity and accuracy of the macroscopic model is successfully checked through the comparison with direct numerical simulations of the initial microscale model for amperometry tests. Predictions obtained from the upscaled model on the current intensity versus the scanning potential during voltammetry reveal to be in very good agreement with experimental results reported in the literature. These results show the capability of the macroscopic model to analyze the behavior of the porous electrode. In particular, it provides an efficient tool to study the dependence of the current intensity on the microstructure of the porous material and on the electrochemical parameters with the perspective of optimizing the electrode efficiency.
\end{abstract}

\section{Introduction}

Porous electrodes have become a privileged solution for miniaturized and potentially in vivo implantable active and passive electro-analytical devices such as biosensors, bioactuators, batteries, etc. thanks to their high specific surface area which allows efficient in situ electrical energy production through the use of rather well-controlled redox couples. Indeed, for a given over-potential, such electrodes may provide electrical current at least one order

* Corresponding author. of magnitude higher than classical flat electrodes of the same size (Walcarius and Kuhn, 2008; Szamocki et al., 2006) making possible a reduction of the overall macroscopic size of electrodes down to a few tens of micrometers in thickness and a few millimeters in extent. For the past two decades, porous electrodes have often been synthesized by self-assembly and electrodeposition (Reculusa and Ravaine, 2003) using the Langmuir-Blodgett technique (Blodgett, 1935). These techniques allow to create a wide range of porous microstructures with tunable porosity as well as controllable architecture (Karajić et al., 2015).

An illustration of the overall procedure, inspired from Bartlett et al. (2000), is sketched in Fig. 1.1 representing the four main steps to obtain a miniaturized two-electrode electrochemical cell using this technique (Karajić et al., 2015). The first step consists in the 


\begin{tabular}{|c|c|c|c|}
\hline \multicolumn{4}{|c|}{ Nomenclature } \\
\hline $\mathrm{A}_{s f}$ & solid/fluid interface in the averaging volume & $n$ & number of transferred electrons \\
\hline$A_{s f}$ & measure of $A_{s f}\left(m^{2}\right)$ & $\mathbf{n}$ & unit normal vector to the solid-fluid interface pointing \\
\hline$A_{f e}$ & $\begin{array}{l}\text { interface between the fluid phase in the electrode and } \\
\text { the external bulk fluid }\end{array}$ & $\mathbf{r}$ & $\begin{array}{l}\text { out of the fluid phase } \\
\text { position vector (m) }\end{array}$ \\
\hline$a_{v}$ & specific area $\left(\mathrm{m}^{-1}\right)$ & $R$ & Ideal gas constant $(\mathrm{J} / \mathrm{K} / \mathrm{mol})$ \\
\hline b & closure variable that maps $\nabla\left\langle c_{A}\right\rangle^{f}$ onto $\tilde{c}_{A}(\mathrm{~m})$ & $r_{0}$ & characteristic size of the averaging volume (m) \\
\hline$c_{X}$ & concentration of species $X(X=A$ or $B)\left(\mathrm{mol} / \mathrm{m}^{3}\right)$ & $r_{E}$ & potential scan rate $(\mathrm{V} / \mathrm{s})$ \\
\hline$c_{A}^{0}$ & bulk concentration of species $A\left(\mathrm{~mol} / \mathrm{m}^{3}\right)$ & $R_{\text {red }}$ & reduction reaction rate $\left(\mathrm{mol} / \mathrm{m}^{2} / \mathrm{s}\right)$ \\
\hline$\left\langle c_{A}\right\rangle$ & superficial average of $c_{A}\left(\mathrm{~mol} / \mathrm{m}^{3}\right)$ & $s$ & closure variable that maps $\left\langle c_{A}\right\rangle^{f}$ onto $\tilde{c}_{A}$ \\
\hline$\left\langle c_{A}\right\rangle^{f}$ & intrinsic average of $c_{A}\left(\mathrm{~mol} / \mathrm{m}^{3}\right)$ & $T$ & temperature $(\mathrm{K})$ \\
\hline $\begin{array}{l}\left\langle c_{A}\right\rangle_{s f} \\
\tilde{c}_{A}\end{array}$ & $\begin{array}{l}\text { area average of } c_{A}\left(\mathrm{~mol} / \mathrm{m}^{2}\right) \\
\text { concentration deviation }\left(\mathrm{mol} / \mathrm{m}^{3}\right)\end{array}$ & $\mathbf{u}$ & $\begin{array}{l}\text { effective vector associated with the chemical reaction } \\
(\mathrm{m} / \mathrm{s})\end{array}$ \\
\hline$\frac{A_{A}}{c_{A}^{*}}$ & $\begin{array}{l}\text { dimensionless concentration averaged over the fluid do- } \\
\text { main }\end{array}$ & $\begin{array}{l}V \\
V\end{array}$ & $\begin{array}{l}\text { averaging volume } \\
\text { measure of } \mathrm{V}\left(\mathrm{m}^{3}\right)\end{array}$ \\
\hline $\begin{array}{l}\mathcal{D}_{A} \\
D_{e f f}\end{array}$ & $\begin{array}{l}\text { molecular diffusion coefficient of species } A\left(\mathrm{~m}^{2} / \mathrm{s}\right) \\
\text { effective diffusion tensor }\left(\mathrm{m}^{2} / \mathrm{s}\right)\end{array}$ & $\mathrm{v}_{f}$ & $\begin{array}{l}\text { domain occupied by the fluid phase within the averag- } \\
\text { ing volume }\end{array}$ \\
\hline $\mathcal{D}_{\text {eff }}$ & $\begin{array}{l}\text { effective diffusion coefficient in the isotropic case } \\
\left(\mathrm{m}^{2} / \mathrm{s}\right)\end{array}$ & $\begin{array}{l}V_{f} \\
V_{s}\end{array}$ & $\begin{array}{l}\text { measure of } V_{f}\left(\mathrm{~m}^{3}\right) \\
\text { domain occupied by the solid phase within the averag- }\end{array}$ \\
\hline$d_{s}, d_{c}$ & $\begin{array}{l}\text { diameter of spherical pore, pore connection window } \\
\text { size }(m)\end{array}$ & $V_{s}$ & $\begin{array}{l}\text { ing volume } \\
\text { measure of } \mathrm{V}_{s}\left(\mathrm{~m}^{3}\right)\end{array}$ \\
\hline$E, E^{0}$ & potential, standard potential (V) & $\mathbf{x}$ & position vector of the centroid of the averaging volume \\
\hline F & Faraday's constant $(\mathrm{C} / \mathrm{mol})$ & & $(\mathrm{m})$ \\
\hline $\begin{array}{l}\mathcal{F}_{A} \\
\mathcal{G}_{A}\end{array}$ & $\begin{array}{l}\text { initial concentration of species } A\left(\mathrm{~mol} / \mathrm{m}^{3}\right) \\
\text { concentration of species } A \text { at the interface } A_{f e}\left(\mathrm{~mol} / \mathrm{m}^{3}\right)\end{array}$ & $\mathbf{y}$ & $\begin{array}{l}\text { relative position vector locating points in the fluid phase } \\
(\mathrm{m})\end{array}$ \\
\hline$i_{v}$ & $\begin{array}{l}\text { current per unit volume at the scale of the representa- } \\
\text { tive unit cell }\left(\mathrm{A} / \mathrm{m}^{3}\right)\end{array}$ & $\begin{array}{l}\mathrm{x}, \mathrm{y}, \mathrm{z} \\
()^{*}\end{array}$ & $\begin{array}{l}\text { directions in cartesian coordinate system } \\
\text { dimensionless quantity }\end{array}$ \\
\hline$I$ & total current intensity $(A)$ & $\alpha$ & electron transfer coefficient \\
\hline i & unit tensor & $\alpha_{A}$ & potential dependent term, $\alpha_{A}=\exp \frac{-\alpha n F\left(E-E^{0}\right)}{R T}$ \\
\hline $\mathcal{I}_{s f}$ & solid/fluid interface & $\nabla$. & divergence operator \\
\hline$j$ & current density $\left(\mathrm{A} / \mathrm{m}^{2}\right)$ & $\varepsilon_{f}$ & porosity of the electrode \\
\hline$k_{0}$ & electron transfer rate constant $(\mathrm{cm} / \mathrm{s})$ & $\Omega$ & domain occupied by the electrode \\
\hline $\begin{array}{l}K_{e f f} \\
K i\end{array}$ & $\begin{array}{l}\text { effective reaction rate coefficient }(\mathrm{m} / \mathrm{s}) \\
\text { pore-scale kinetic number, } K i=\frac{k_{0} \alpha_{A} \ell_{P}}{\bar{A}}\end{array}$ & $\Omega_{e}$ & $\begin{array}{l}\text { domain occupied by the external bulk fluid (Nernstian } \\
\text { layer) }\end{array}$ \\
\hline$L_{\text {elec }}$ & electrode thickness $(\mathrm{m})$ & $\Omega_{f}$ & domain occupied by the fluid phase within the electrode \\
\hline$L_{N}$ & Nernstian layer thickness $(\mathrm{m})$ & $\Omega_{s}$ & domain occupied by the solid phase within the elec- \\
\hline$\ell_{i}$ & periodic lattice vector in the $i$ th-direction (m) & & tos \\
\hline$l_{p}$ & characteristic pore size $\left(\ell_{p} \equiv d_{s}\right)(\mathrm{m})$ & $\nabla$ & gradient operator \\
\hline$\ell_{R E V}$ & size of the periodic representative unit cell (m) & & \\
\hline
\end{tabular}

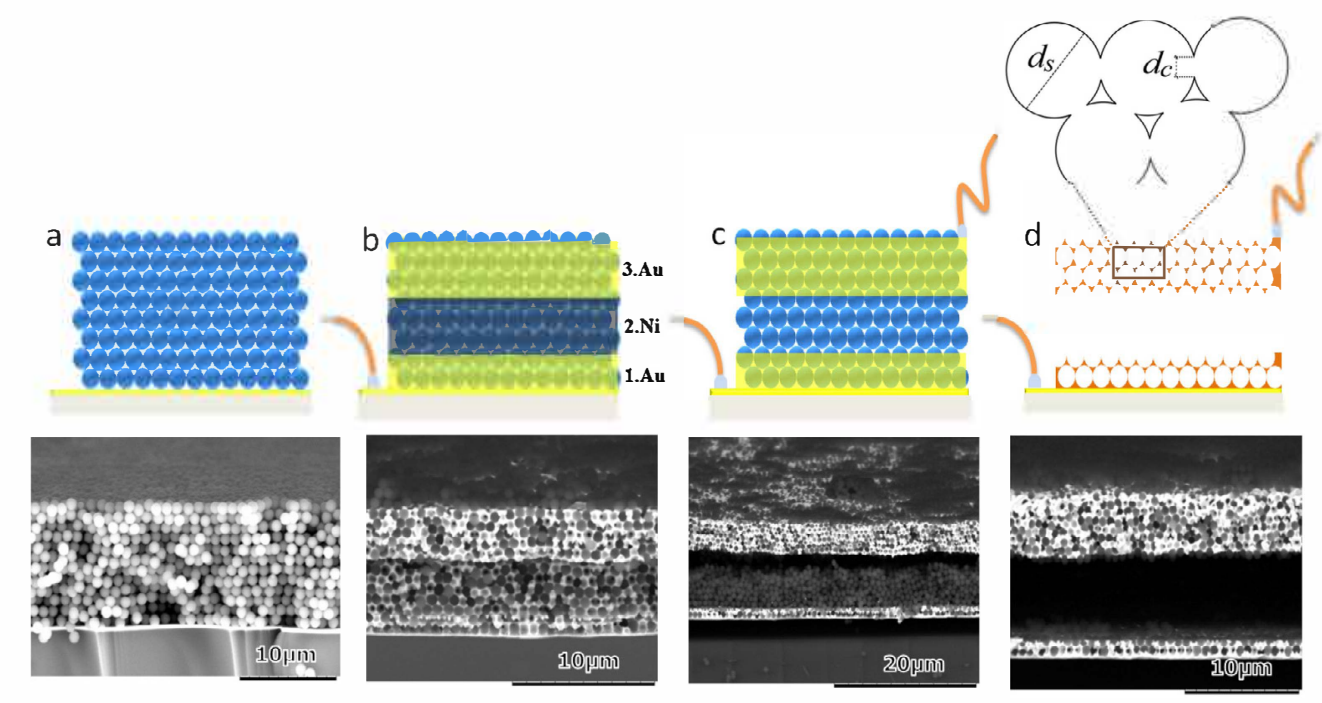

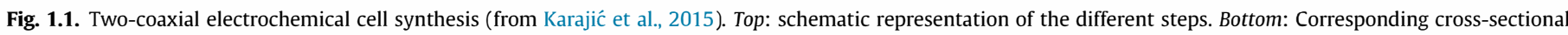
SEM images of the material. 
deposition of a colloidal template of self-assembled silica beads, with a diameter $d_{s}$ and a number of layers that can be adjusted, on a gold-coated glass substrate (Reculusa and Ravaine, 2003; Szamocki et al., 2006) (see Fig. 1.1a). Secondly, sequential electrodeposition of gold (or other conducting materials like polypyrrol (Heim et al., 2012)) and nickel layers is performed so that the limits between these layers, and hence their thickness, can be strictly controlled (Reculusa et al., 2011) (Fig. 1.1b). Finally, the nickel layer and the silica template are successively dissolved to obtain a two-electrode device (Fig. 1.1c and d). It should be noted that the purpose of the nickel layer is to form an electrical insulation between the two electrodes and the thickness of this layer may be typically of few beads diameters $d_{s}$. The resulting porous structure is such that, due to the limitation of the electrodeposition process in the interstice between touching beads, open connecting windows of characteristic size $d_{c}$ between two adjacent pores remain. Evidently, the mass and electron transfer within the porous structure, as well as the reactive mechanism governing the whole process that conditions the resulting efficiency of the electrode, are intimately related to the architecture of the porous material.

As indicated above, current available methods for electrode engineering allow for rather well-controlled micro- or nanostructured porous materials with a given pore-size gradient and distribution to be achieved. However, the question of how an optimal pore architecture can be designed to maximize the electrode power efficiency has been receiving an empirical answer so far. A rational approach is indeed crucial in order to clearly relate the current available at the macroscopic level to the microsctructure on the basis of a careful physical description of mass and electron transfer coupled to reaction mechanisms at play at the microscale (pore-scale) and their implication at the macroscale. So far, modeling has been often restricted to a 1D approach, making use, most of the time, of empirical macroscopic models (Barnes et al., 2014; Do et al., 2014). A formal upscaled model, obtained from volume averaging and describing the transport and reaction process in a porous electrode, taking into account three phases (solid, liquid and gas), was proposed two decades ago (Vidts and White, 1997). However, there is no closure scheme available in this work so that effective parameters in the macroscopic equations can not be properly estimated. In Ferguson and Bazant (2012), correlations between the effective diffusivity (or conductivity) and the tortuosity were employed, based on Wiener or Hashin-Shtrikman limiting bounds, without any explicit dependence on the real geometry of the microstructure. In Ender (2015), a homogenized porous electrode model for lithium/ion cell electrode was proposed where the effective conductivity was directly obtained from the experimental measurement.

Our purpose in the present study is to contribute to a physically sound multi-scale modeling of the coupled transfer and reactions in a simplified case where exchange of electrons directly occurs between the species in the fluid and the pore surfaces without any contribution of enzymes or redox mediator. As a proof of concept, the reduction of $\mathrm{O}_{2}$ to $\mathrm{H}_{2} \mathrm{O}_{2}$ has been chosen. In this context, at the pore-scale, mass transfer is essentially diffusive, while a cathodic oxygen reduction-like reaction can be considered making use of the Butler-Volmer model (Butler, 1924; Butler, 1932). Starting from the pore-scale initial boundary-value problem, the method of volume averaging (Whitaker, 1999) is employed in order to derive a closed macroscopic mass transfer equation containing macroscopic effective coefficients together with the expression of the current available at the electrode. The effective mass transport coefficient is obtained from the solution of a closure problem that allows passing the microscale information up to the macroscopic level.
The article is organized as follows. The initial boundary value problem is formulated at the microscopic scale in Section 2, a numerical solution of which is provided on model porous structures in Section 3. With the aim of deriving a model that operates at the macroscale and that is much less demanding in terms of computational resources while keeping the essential information from the pore-scale, the upscaling is proposed in Section 4. This yields an effective model that allows the determination of the effective current available at the electrode. In Section 5, numerical solutions of the macroscopic upscaled model are reported for the different microstructures under consideration, characterized by their pore diameter $d_{s}$, pore connection window size $d_{c}$, porosity $\varepsilon_{f}$, specific surface area $a_{v}$, and macroscopic geometries, defined by the electrode thickness $L_{\text {elec }}$ and fluid external diffusion layer (Nernstian layer) thickness $L_{N}$. Successful comparisons with experimental data are also reported in this section. Conclusive remarks are drawn in Section 6.

\section{Microscopic pore-scale model}

The porous electrode, immersed in the reactive solution, occupies the spatial domain $\Omega$ composed of the solid skeleton in $\Omega_{s}$ and the fluid phase in $\Omega_{f}$, the interface between the two being denoted by $\mathcal{I}_{s f}=\Omega_{f} \cap \Omega_{s}$ where redox reaction takes place (Fig. 2.1). Here, we focus on the direct reduction reaction of oxygen at the cathode without any catalysis, so that the electrochemical process can be represented by Barnes et al. (2014)

$A+n e^{-} \stackrel{k_{0}}{\rightarrow} B \quad \mathbf{r} \in \mathcal{I}_{s f}$

where species $A$ and $B$ may represent, for instance, oxygen and hydrogen peroxide respectively. We have denoted by $\mathbf{r}$ the position vector on $\mathcal{I}_{s f}$ locating the sites where $n$ electrons are captured by the acceptor $A$ whereas $k_{0}$ is the electron transfer rate constant. Since the interest is laid upon reduction, it is sufficient to specify the mass balance for species $A$ which diffuses from the external bulk fluid $\Omega_{e}$ in the Nernstian diffusion layer towards the electrode external surface, and then through the pore space $\Omega_{f}$ towards the pore walls $\mathcal{I}_{s f}$ (Fig. 2.1) where electrons transfer takes place. The Nernstian layer in the vicinity of the electrode's external surface represents the region where a significant concentration gradient occurs in the bulk fluid.

In practice, $A$ is sufficiently diluted in the supporting electrolyte for mass transfer to be described as a Fickian process (Fick, 1995). The Initial Boundary Value Problem (IBVP) governing the diffusionreaction mechanism within the electrode at the pore-scale can hence be written as

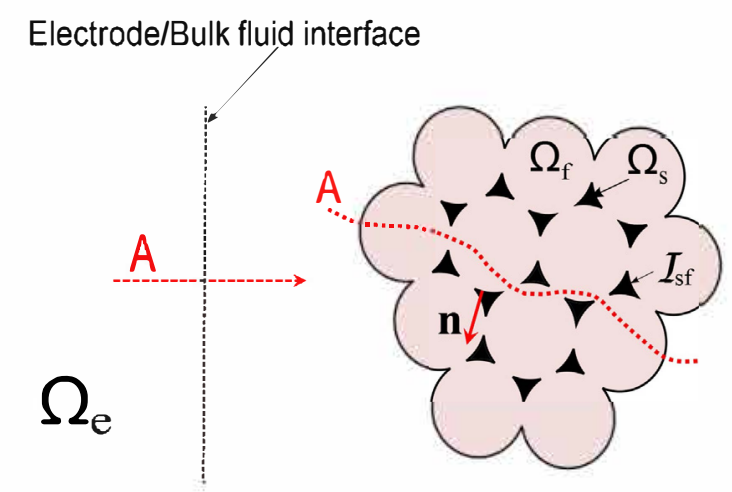

Fig. 2.1. Pore-scale configuration with the solid and fluid domains, their interface and a diffusive path of species $A$. 
$\frac{\partial c_{A}}{\partial t}=\nabla \cdot\left(\mathcal{D}_{A} \nabla c_{A}\right) \quad$ in $\Omega_{f}$

B.C.1 $-\mathbf{n} \cdot \mathcal{D}_{A} \nabla c_{A}=-R_{\text {red }} \quad$ at $\mathcal{I}_{\text {sf }}$

I.C. $c_{A}=\mathcal{F}_{A}(\mathbf{r}) \quad \mathbf{r} \in \Omega_{f}, \mathrm{t}=0$

B.C.2 $c_{A}=\mathcal{G}_{A}(\mathbf{r}, t) \quad \mathbf{r} \in \mathrm{A}_{\mathrm{fe}}, \forall \mathrm{t}$

where $c_{A}=c_{A}(\mathbf{r}, t)$ and $\mathcal{D}_{A}$ are the concentration at $\mathbf{r}$ and $t$ and molecular diffusion coefficient of species $A$, respectively, while $R_{\text {red }}$ is the reduction reaction rate, and $\mathbf{n}$ the normal unit vector at the fluid solid interface pointing out of the fluid phase. In the expression of the external boundary condition B.C.2, $A_{f e}=\Omega_{f} \cap \Omega_{e}$ is the entrance and/or exit boundary of the fluid phase $\Omega_{f}$ from/into the external bulk fluid $\Omega_{e}$. In our case, the reacting molecule (oxygen) is not charged and is a dissolved species in the liquid in contact with the electrode, so, for the sake of simplicity, adsorption/desorption mechanisms are not taken into account in the present analysis.

The reduction reaction rate, $R_{\text {red }}$, is related to the corresponding microscopic electrical current density, $j$, from Faraday's law (Faraday, 1833) according to

$R_{\text {red }}=\frac{j}{n F}$

Moreover, the relationship between the current density and the potential, $E$, is classically described by the Butler-Volmer equations, which, in the general case is expressed as (Butler, 1924; Butler, 1932)

$j=n k_{0} F\left[\exp \left(\frac{(1-\alpha) n F\left(E-E^{0}\right)}{R T}\right) c_{B}-\exp \left(\frac{-\alpha n F\left(E-E^{0}\right)}{R T}\right) c_{A}\right]$

In this expression, $\alpha$ is the electron transfer coefficient ( $\alpha$ takes values of about 0.5 ), $E^{0}$ the standard potential, $R$ and $F$ are the ideal gas and Faraday's constants respectively, $T$ is the temperature and $c_{B}$ represents the concentration of species $B$ resulting from the reduction of species $A$. In the particular case of simple oxygen reduction, the oxidation reaction can be completely ignored so that the first exponential term on the right hand side of Eq. (4) can be reasonably neglected. Under these circumstances, Eq. (4) can be simplified to the Tafel equation (Tafel, 1905) given by

$\begin{aligned} j & =-n k_{0} F \exp \left(\frac{-\alpha n F\left(E-E^{0}\right)}{R T}\right) c_{A} \\ & =-n k_{0} F \alpha_{A} c_{A} \text { at } \mathcal{I}_{s f}\end{aligned}$

where $\alpha_{A}$ is a potential dependent parameter which expression is $\alpha_{A}=\exp \frac{-\alpha n F\left(E-E^{0}\right)}{R T}$. Hence,

$R_{\text {red }}=-k_{0} \alpha_{A} c_{A} \quad$ at $\mathcal{I}_{\text {sf }}$
For a given structure, geometry and operating conditions, the IBVP defined in Eqs. (2) and (6) can be solved directly in order to finally compute the current available at the electrode given by

$I=-n k_{0} F \alpha_{A} \int_{\mathcal{I}_{s f}} c_{A} \mathrm{~d} A$

\section{Numerical solution of the microscopic model}

In practice, $\mathcal{G}_{A}(\mathbf{r}, t)$ in Eq. (2d) is usually unknown at the interface with the bulk fluid and the boundary condition B.C.2 shall be replaced by considering the Nernstian diffusion layer, $\Omega_{e}$, of thickness $L_{N}$, out of which $c_{A}$ remains constant, i.e. $c_{A}=c_{A}^{0}$.

Direct numerical simulations (DNS) were carried out by solving the 3D microscopic pore-scale model described in Section 2. Several arrangements representative of the porous electrode microstructure may be considered such as cubic (C), bodycentered cubic (BCC) and face-centered cubic (FCC) with periodic unit cells, of size $\ell_{R E V}$, represented in Fig. 3.1. Characteristics of these microstructures are imposed by the pore diameter $d_{s}$ and the connection window size $d_{c}$ between two adjacent pores from which the porosity and specific area can be determined as will be further discussed in Section 5.1. The FCC structure, which is the most compact one, was used in the pore-scale simulations as it realistically represents a homogeneous template as the one reported in Fig. 1.1 (Reculusa et al., 2011).

Simulations, which correspond to chronoamperometric 3D numerical experiments, were performed on a computational domain represented in Fig. 3.2. It is composed of the porous electrode, between $z=-L_{\text {elec }}$ and $z=0$, and the Nernstian fluid layer between $z=0$ and $z=L_{N}$ where molecular diffusion, governed by Eq. (2a), takes place. The electrode is made of an array of $1 \times 1 \times \frac{L_{\text {elec }}}{\ell_{\text {REV }}}$ unit cells in the $\mathrm{x}, \mathrm{y}$ and $\mathrm{z}$-directions respectively. Periodic boundary conditions are applied along $x$ and $y$ with the idea that the electrode is either of infinite extension in the $x-y$ plane or has an annular shape with its mean radius much larger than its thickness (i.e. is quasi-plane) and its length much larger than $\ell_{R E V}$ for the periodic boundary condition in the axial direction to be relevant. The additional boundary conditions are a zero diffusive flux at the lower boundary of the electrode, $z=-L_{\text {elec }}$, and constant concentration, $c_{A}^{0}$, at the upper boundary of the Nernstian layer, $z=L_{N}$. The initial concentration is supposed to be uniform equal to $c_{A}^{0}$.

Using $\ell_{R E V}, \frac{\ell_{R E V}^{2}}{\mathcal{D}_{A}}$ and $c_{A}^{0}$ as the reference length, time and concentration and denoting dimensionless quantities with the superscript ${ }^{*}$, the dimensionless problem for the chronoamperometric numerical experiment is given by

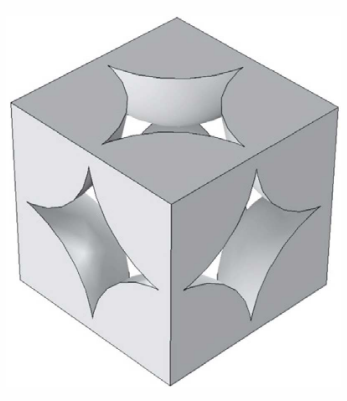

C

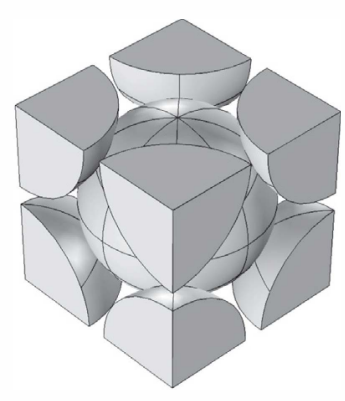

$\mathrm{BCC}$

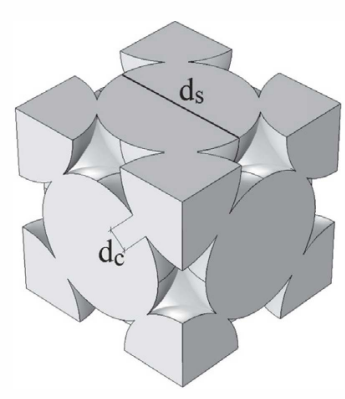

FCC

Fig. 3.1. Periodic unit cells of the three arrangements considered for the porous electrode microstructures: C, BCC and FCC. 


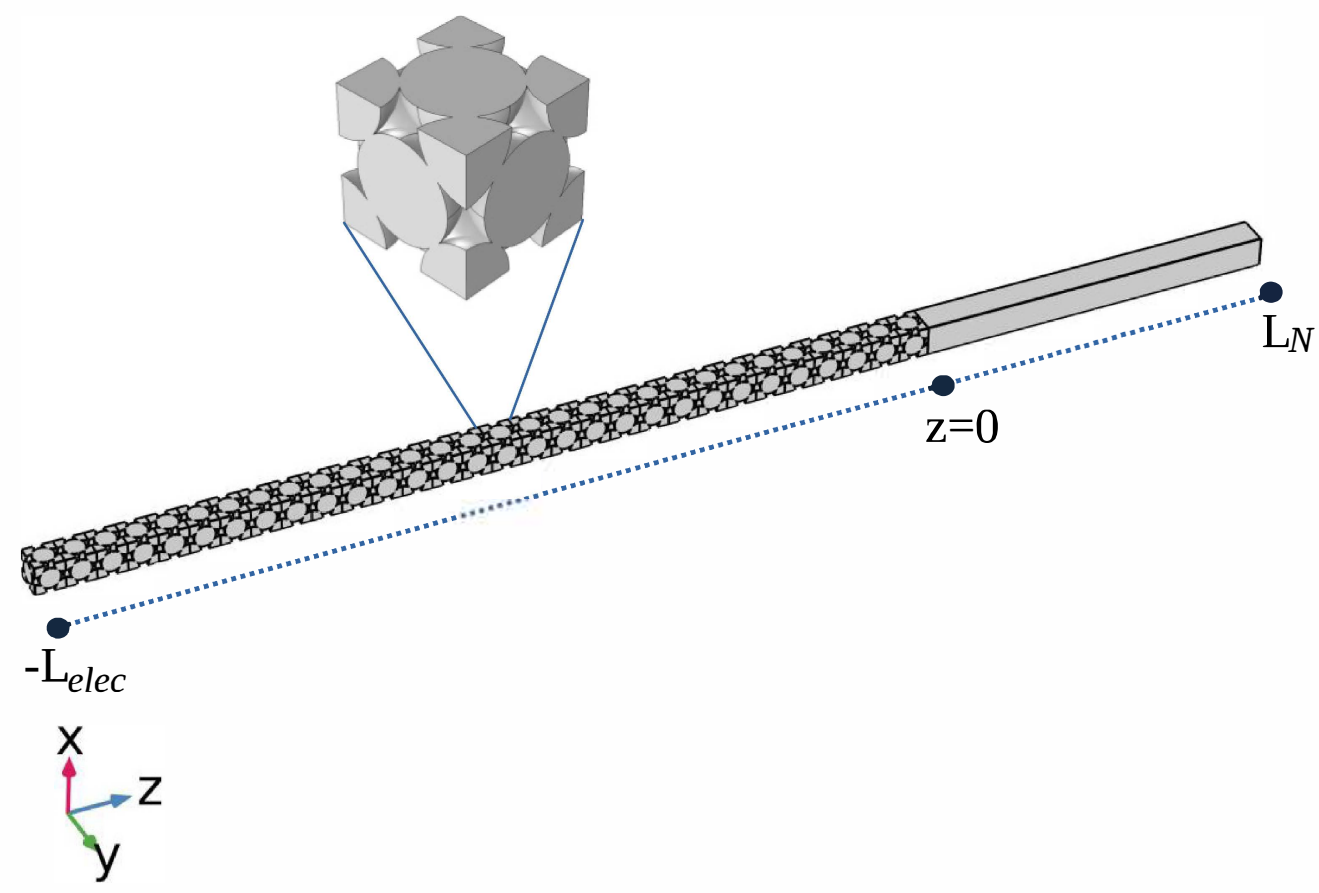

Fig. 3.2. 3D domain for the DNS.

$\frac{\partial c_{A}^{*}}{\partial t^{*}}=\nabla^{* 2} c_{A}^{*} \quad$ in $\Omega_{f} \cup \Omega_{e}$

B.C.1 $-\mathbf{n} \cdot \nabla^{*} c_{A}^{*}=\frac{k_{0} \alpha_{A} \ell_{R E V}}{\mathcal{D}_{A}} c_{A}^{*} \quad$ at $\mathcal{I}_{s f}$

B.C. $2-\mathbf{n} \cdot \nabla^{*} c_{A}^{*}=0 \quad$ at $z^{*}=-L_{\text {elec }}^{*}$

B.C. $3 c_{A}^{*}=1 \quad$ at $z^{*}=L_{N}^{*}$

I.C. $c_{A}^{*}=1 \quad$ in $\Omega_{f} \cup \Omega_{e}$ at $t^{*}=0$

For completeness, periodic boundary conditions on both the concentration and flux in the $\mathrm{x}$ and $\mathrm{y}$ directions must be added to the above set of equations. The reference length $\ell_{R E V}$ used in the above dimensionless form is only a choice and does not necessarily correspond to the relevant physical scaling which would rather impose $d_{s}$ as the reference length. This choice was made and will be kept for the 1D macroscopic model in Section 5 in order to perform a direct comparison on concentration and current versus dimensionless space and time variables.

To illustrate the solution, simulations were carried out with $L_{\text {elec }}^{*}=30$ and $L_{N}=20 \mu \mathrm{m}\left(L_{N}^{*} \simeq 12\right)$. In practice, the value of $L_{N}$ is difficult to estimate accurately and the latter value is just a choice to illustrate the solution. Values of the other physical parameters used for the simulations are indicated in Table 3.1. These parameters were chosen to be consistent with the experimental configuration further considered in Section 5.3. In particular, the standard potential was taken as the one for the reduction represented in Eq. (1) relative to the $\mathrm{Ag} / \mathrm{AgCl}$ reference electrode. Since the standard potential for the reaction (1) relative to the standard hydrogen electrode (SHE) is $0.67 \mathrm{~V}$ whereas the potential difference of the $\mathrm{Ag} / \mathrm{AgCl}$ electrode and SHE is $0.22 \mathrm{~V}$, this leads to $E^{0}=0.45 \mathrm{~V}$ in our case. In addition, it must be noted that the overall electron transfer results from a complex succession of elementary steps which detailed model is still a subject of debate, in particular for oxygen reduction (Shinagawa et al., 2015). In this latter case, there exist a pseudo-elementary rate determining step, involving a single electron transfer, even though the overall reduction occurs with two electrons (Zhang, 2008). For this reason, the value of $n$ can be rather taken to be equal to 1 in the exponential term of the driving force in the Butler-Volmer Eq. (5). It should be recalled that the
Table 3.1

Parameters used in the simulations.

\begin{tabular}{cccc}
\hline Parameter & Symbol & Value & Unit \\
\hline Ideal gas constant & $R$ & 8.314 & $\mathrm{~J} /(\mathrm{mol} \mathrm{K})$ \\
Faraday's constant & $F$ & 96485.33 & $\mathrm{C} / \mathrm{mol}$ \\
Number of electron transferred & $n$ & 2 & - \\
Electron transfer coefficient & $\alpha$ & 0.66 & - \\
Standard potential vs. $E_{A g / A g C l}^{0}$ & $E_{0}$ & 0.45 & $\mathrm{~V}$ \\
Temperature & $T$ & 300 & $\mathrm{~K}$ \\
Potential vs. $E_{A g / A g C l}$ & $E$ & 0.1 & $\mathrm{~V}$ \\
Bulk concentration & $c_{A}^{0}$ & 1.2 & $\mathrm{~mol} / \mathrm{m}^{3}$ \\
Diffusion coefficient & $\mathcal{D}_{A}$ & $2 \times 10^{-9}$ & $\mathrm{~m}^{2} / \mathrm{s}$ \\
Spherical pore diameter & $d_{s}$ & 1.2 & $\mu \mathrm{m}$ \\
Relative pore connection window size & $d_{C} / d_{S}$ & $15 \%$ & - \\
Size of the periodic unit cell & $\ell_{R E V}$ & 1.678 & $\mu \mathrm{m}$ \\
\hline
\end{tabular}

value of $L_{\text {elec }}^{*}$ represents the number of unit cells composing the electrode in the $z$-direction.

The software COMSOL Multiphysics was used to solve this problem. Careful attention was paid to the mesh convergence and, to fulfill this requirement in the configuration defined above, we used a physics-controlled mesh including extremely fine grid blocks composed of $1.310^{7}$ tetrahedral elements in the overall domain represented in Fig. 3.2.

It should be noted that the value of $k_{0}$ can be extremely small (down to less than $10^{-9} \mathrm{~cm} / \mathrm{s}$ ) when the electrode surface is not catalyzed by any enzymes or electrons are not transferred by a mediator (Renslow et al., 2011). Hence, in our case, the investigated range of $k_{0}$ is from $10^{-7} \mathrm{~cm} / \mathrm{s}$ to $10^{-9} \mathrm{~cm} / \mathrm{s}$.

Dimensionless concentration fields obtained from DNS in $\Omega_{f}$ and $\Omega_{e}$ are represented in Fig. 3.3 at dimensionless times $t^{*}=10,100$ and 200 for $k_{0}=10^{-8} \mathrm{~cm} / \mathrm{s}$ clearly highlighting the coupled diffusion-reaction process inside the porous electrode. In particular, it shows that the portion of the electrode, where the concentration gradient (i.e. the mass flux) is strong enough to supply the reaction, remains of relatively small extent (about $1 / 4$ th of the total electrode length at $t^{*}=200$ for instance) in the region 

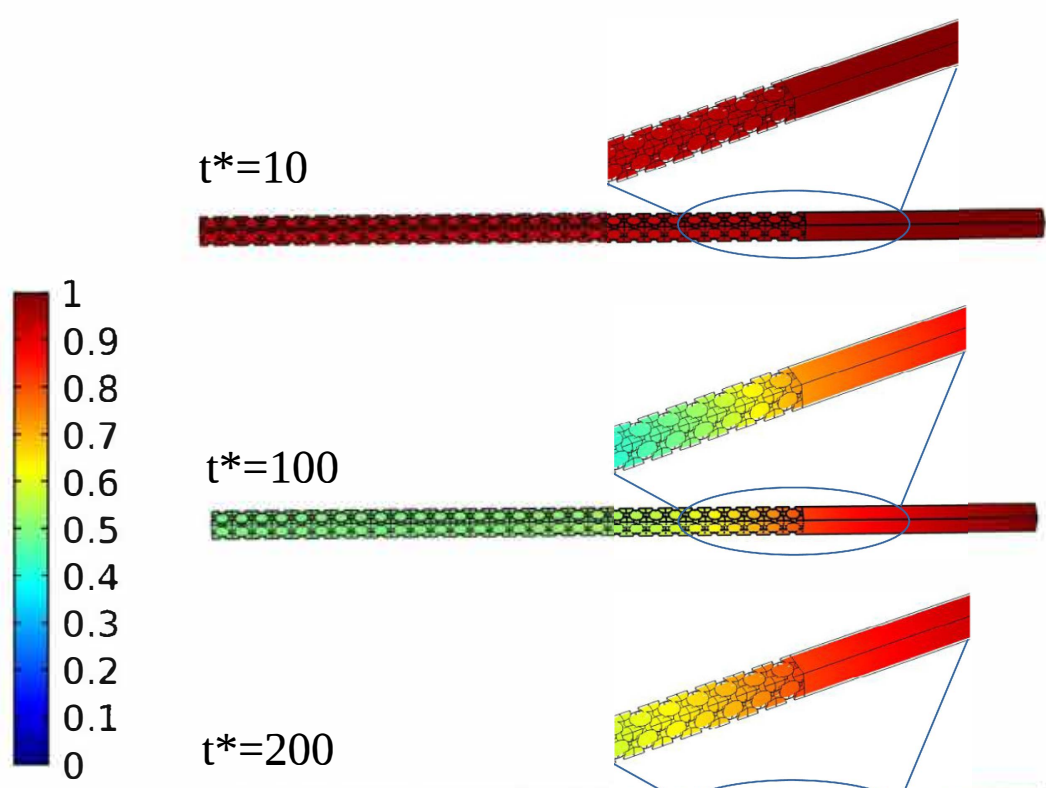

0.4

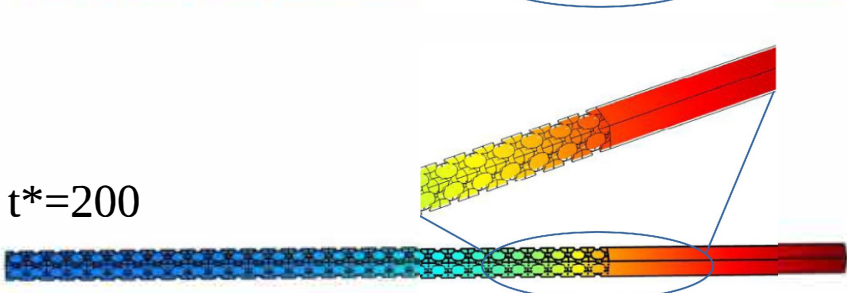

Fig. 3.3. Dimensionless concentration fields $c_{A}^{*}$ at $t^{*}=10,100,200$ for $k_{0}=10^{-8} \mathrm{~cm} / \mathrm{s}$.

close to $z=0$. The remaining part of the electrode operates in a mass-transfer limited regime.

To analyze the concentration evolution, the dimensionless concentration averaged over the fluid domain $\Omega_{f}$, denoted by $\overline{C_{A}^{*}}$ and defined by

$\overline{c_{A}^{*}}=\frac{1}{\left|\Omega_{f}\right|} \int_{\Omega_{f}} c_{A}^{*} \mathrm{~d} V$

is represented versus $t^{*}$ in Fig. 3.4 for three different values of the electron transfer rate constant, $k_{0}$. This figure shows that $\overline{c_{A}^{*}}$ strongly decreases at the early stage of the experiment especially when $k_{0}$ is large. This is due to the initial condition $c_{A}^{*}=1$ which provides a large reduction reaction rate at the solid-fluid interfaces everywhere in the pore space while, as indicated above, the region far from the Nernstian layer remains poorly supplied with reactant over time. A plateau is finally obtained after a sufficiently long time

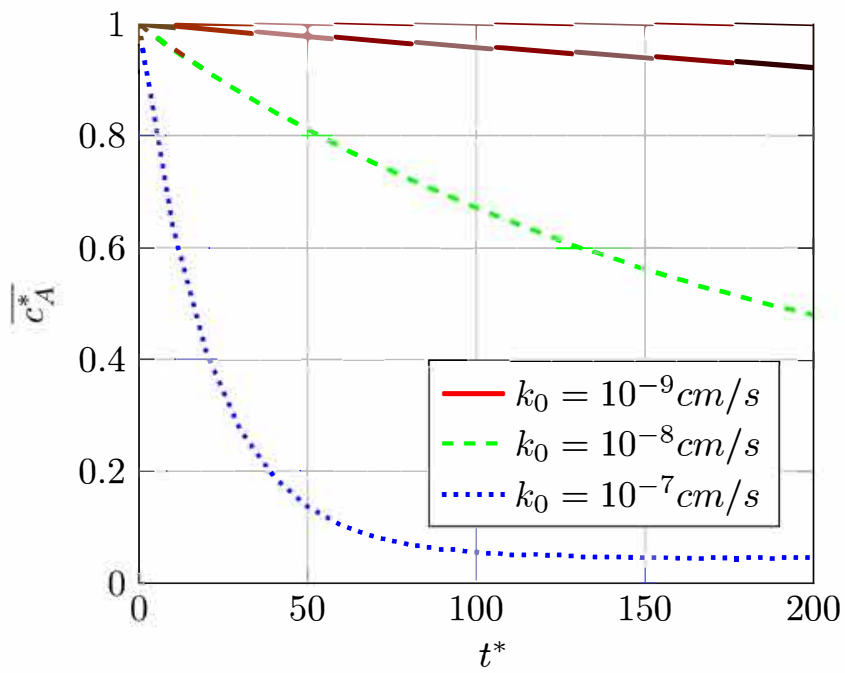

Fig. 3.4. Dimensionless dynamics of the averaged dimensionless concentration $\overline{c_{A}^{*}}$ for three different values of $k_{0}$. corresponding to the steady state where the diffusive flux of species $A$ entering the electrode is balanced by the reaction at the pore surfaces. As expected, the larger $k_{0}$, the stronger the rate of decrease of $\overline{c_{A}^{*}}$ and the shorter the steady-state to be reached.

In addition, the current available may be computed from the expression in Eq. (7). This numerical result will be shown and discussed in Section 5.2 when compared with the current obtained from the macroscopic model.

While DNS are able to provide detailed and accurate results, the important computational time and resources they require (typically $52 \mathrm{~min}$ for one simulation in the above-mentioned conditions on a Dell PowerEdge 430-2 processors Intel Xeon E5-2630v3) are such that they can not be reasonably envisaged if one is willing to carry out a thorough analysis of the macroscopic current-tomicrostructure relationship. Alternatively, an upscaling procedure can be applied to the above equations in order to obtain a macroscopic description of the coupled diffusion-reaction process in the porous medium and an expression of the current at the macroscale. This is the objective of the following section.

\section{Macroscopic effective model}

The IBVP under consideration given in Eqs. (2) corresponds to a diffusion with heterogeneous reaction, and resembles the one for which upscaling is detailed in Whitaker (1999) for a homogeneous porous medium using the volume averaging method. For the sake of brevity, the main result of the development is recalled with the necessary details reported in Appendix A.

The upscaling procedure starts with the definition of an averaging domain $\vee$ (of measure $V$ and size $r_{0}$ ) including the solid and fluid domains $\vee_{s}$ and $\vee_{f}$ (of measure $V_{s}$ and $V_{f}$ respectively) and the solid fluid interface $A_{s f}$ (of measure $A_{s f}$ ) from which the porosity, $\varepsilon_{f}$, and specific area, $a_{v}$, can be obtained as

$\varepsilon_{f}=\frac{V_{f}}{V}$

$a_{v}=\frac{A_{s f}}{V}$ 
Three different averaging operators are used to carry out the upscaling, namely the superficial, intrinsic and area averages respectively given by

$$
\begin{aligned}
\left.\left\langle c_{A}\right\rangle\right|_{\mathbf{x}} & =\left.\frac{1}{V} \int_{\mathrm{V}_{f}(\mathbf{x})} c_{A}\right|_{\mathbf{x}+\mathbf{y}} d V \\
\left.\left\langle c_{A}\right\rangle^{f}\right|_{\mathbf{x}} & =\left.\frac{1}{V_{f}} \int_{V_{f}(\mathbf{x})} c_{A}\right|_{\mathbf{x}+\mathbf{y}} d V \\
\left.\left\langle c_{A}\right\rangle_{s f}\right|_{\mathbf{x}} & =\left.\frac{1}{A_{s f}} \int_{A_{s f}(\mathbf{x})} c_{A}\right|_{\mathbf{x}+\mathbf{y}} d A
\end{aligned}
$$

with the straightforward relationship

$$
\left\langle c_{A}\right\rangle=\varepsilon_{f}\left\langle c_{A}\right\rangle^{f}
$$

In these equations, $\mathbf{x}$ is used to locate the centroid of the averaging volume while $\mathbf{y}=\mathbf{r}-\mathbf{x}$ locates any point within $\mathrm{V}_{f}$ relative to $\mathbf{x}$ (see Fig. 4.1), and we shall drop this subscript, unless necessary. The derivation of the upscaled model is subject to a scale hierarchy defined by $\ell_{p} \ll r_{0} \ll L, \ell_{p}$ being the characteristic pore size and $L$ the macroscopic size of the electrode. In the case of the configuration of Fig. 3.2 for instance, $\ell_{p} \equiv d_{s}, r_{0} \equiv \ell_{R E V}$ and $L \equiv L_{\text {elec }}$. All through the article, this scale hierarchy is supposed to be satisfied. The upscaling procedure is carried out according to the four main steps reported in Appendix $\mathrm{A}$ and, under the constraints on the kinetic number $K i=\frac{k_{0} \alpha_{A} \ell_{p}}{\mathcal{D}_{A}} \ll 1$ and time scale $\frac{\mathcal{D}_{A} t}{l_{p}^{2}} \gg 1$, yields the following macroscale diffusion/reaction equation

$\varepsilon_{f} \frac{\partial\left\langle c_{A}\right\rangle^{f}}{\partial t}=\nabla \cdot\left(\varepsilon_{f} \mathbf{D}_{e f f} \cdot \nabla\left\langle c_{A}\right\rangle^{f}\right)-k_{0} \alpha_{A} a_{v}\left\langle c_{A}\right\rangle^{f}$

with the effective diffusion tensor $\mathbf{D}_{\text {eff }}$ given by

$\mathbf{D}_{\text {eff }}=\mathcal{D}_{A}\left(\mathbf{I}+\frac{1}{V_{f}} \int_{\mathrm{A}_{\text {sf }}} \mathbf{n b} \mathbf{b}\right)$

where $\mathbf{b}$, having the dimension of a length, is the solution of the intrinsic closure problem given by (see Appendix A for more details)

$\nabla^{2} \mathbf{b}=0 \quad$ in $V_{f}$

$\mathbf{n} \cdot \nabla \mathbf{b}=-\mathbf{n} \quad$ at $A_{s f}$

$\langle\mathbf{b}\rangle=0$

$\mathbf{b}(\mathbf{r})=\mathbf{b}\left(\mathbf{r}+\ell_{i} \mathbf{e}_{i}\right)$

Eq. (14) operates at scale $L$ once $\varepsilon_{f}, a_{v}$ and $\mathbf{D}_{\text {eff }}$ are computed over a representative unit cell of the structure under concern. When associated to macroscopic boundary conditions, this equation may be solved on the effective medium yielding the field of $\left\langle c_{A}\right\rangle^{f}$ over $\Omega$.

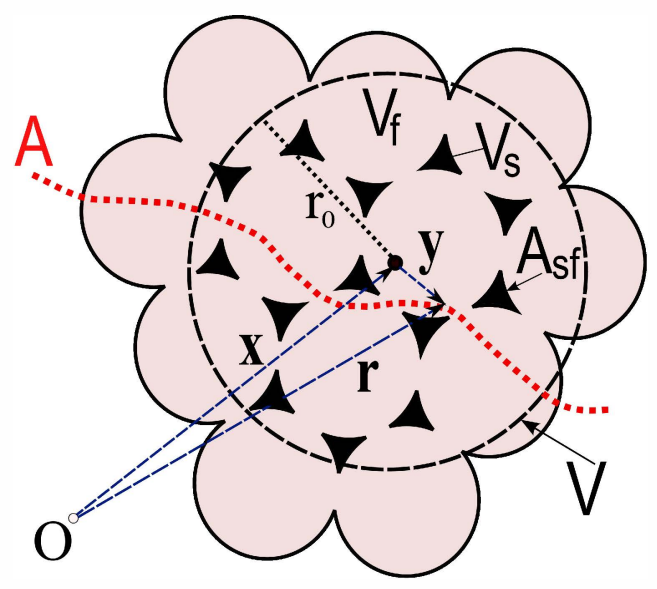

Fig. 4.1. Averaging domain for a two-phase system.
From this solution, the interest is now to obtain the available current at the electrode. To do so, one can start with the expression of the current density at the scale of a representative unit cell of the porous medium, which, by making use of Eq. (5) is given by

$\langle j\rangle_{s f}=\frac{-n k_{0} F \alpha_{A}}{A_{s f}}\left(\int_{\mathrm{A}_{s f}}\left\langle c_{A}\right\rangle^{f} \mathrm{~d} A+\int_{\mathrm{A}_{s f}} \tilde{c}_{A} \mathrm{~d} A\right)$

where we have used the decomposition on $c_{A}$ given in Eq. (A.4). As shown in the development in Step 2 of Appendix A, the second area integral term on the right hand side of the above expression can be neglected whenever the constraint on the kinetic number $\frac{k_{0} \alpha_{A} \ell_{p}}{\mathcal{D}_{A}} \ll 1$ is satisfied. In addition, introducing the Taylor expansion of Eq. (A.10) into the remaining area integral term and using orders of magnitude estimates leads to

$\langle j\rangle_{s f}=-n k_{0} F \alpha_{A}\left\langle c_{A}\right\rangle^{f}$

The current per unit volume, $i_{v}$, at the scale of the representative unit cell is hence given by

$i_{v}=\frac{\langle j\rangle_{s f} A_{s f}}{V}=a_{v}\langle j\rangle_{s f}$

from which the total current available at the electrode is obtained as

$I=\int_{\Omega} i_{\nu} \mathrm{d} V=-n k_{0} F \alpha_{A} a_{v} \int_{\Omega}\left\langle c_{A}\right\rangle^{f} \mathrm{~d} V$

The accuracy of this result associated to the macroscopic model will be checked in Section 5.2 by a direct comparison with the current values computed from Eq. (5) in the DNS approach.

It should be noted that the upscaling approach does not make any assumption on the porous structure itself and can hence be applied to any complex structure provided length-scales separation is satisfied. In particular, it could be envisaged on real images obtained from tomography which may require a local geometrical modification of the structure to make it periodic over the REV. However, this modification would occur over a distance of typical length-scale $\ell_{p}$ and would not significantly impact the values of the effective quantities.

\section{Numerical simulations using the macroscale effective model}

In this section, numerical simulation results obtained with the macroscopic model detailed above are presented and comparisons are carried out both with results of DNS at the pore-scale and experimental data reported in Reculusa et al. (2011).

The porous electrode being now considered as an effective medium, the problem to be solved, written in its dimensionless form, is given by

$$
\begin{aligned}
& \frac{\partial\left\langle c_{A}^{*}\right\rangle^{f}}{\partial t^{*}}=\nabla^{*}\left(\frac{\mathbf{D}_{\text {eff }}}{\mathcal{D}_{A}} \nabla^{*}\left\langle c_{A}^{*}\right\rangle^{f}\right)-\frac{k_{0} a_{v} \ell_{r e v}^{2} \alpha_{A}}{\mathcal{D}_{A} \varepsilon_{f}}\left\langle c_{A}^{*}\right\rangle^{f} \quad \text { in } \Omega \\
& \frac{\partial c_{A}^{*}}{\partial t^{*}}=\nabla^{* 2} c_{A}^{*} \quad \text { in } \Omega_{e} \\
& \text { B.C. } 1 \quad\left\langle c_{A}^{*}\right\rangle^{f}=c_{A}^{*} \quad \text { at } z^{*}=0 \\
& \text { B.C.2 } \mathbf{e}_{z} \cdot \varepsilon_{f} \mathbf{D}_{\text {eff }} \cdot \nabla^{*}\left\langle c_{A}^{*}\right\rangle^{f}=\mathcal{D}_{A} \mathbf{e}_{z} \cdot \nabla^{*} c_{A}^{*} \quad \text { at } z^{*}=0 \\
& \text { B.C.3 }-\mathbf{n} \cdot \mathbf{D}_{\text {eff }} \cdot \nabla^{*}\left\langle c_{A}^{*}\right\rangle^{f}=0 \quad \text { at } z^{*}=-L_{\text {elec }}^{*} \\
& \text { B.C. } 4 c_{A}^{*}=1 \quad \text { at } z^{*}=L_{N}^{*} \\
& \text { I.C. } 1\left\langle c_{A}^{*}\right\rangle^{f}=1 \quad \text { in } \Omega \text { at } t^{*}=0 \\
& \text { I.C. } 2 c_{A}^{*}=1 \quad \text { in } \Omega_{e} \text { at } t^{*}=0
\end{aligned}
$$

where lengths, time and concentrations are again made dimensionless by $\ell_{R E V}, \frac{\ell_{R E V}^{2}}{\mathcal{D}_{A}}$ and $c_{A}^{0}$ respectively, as in Section 3 . Here, it should be 


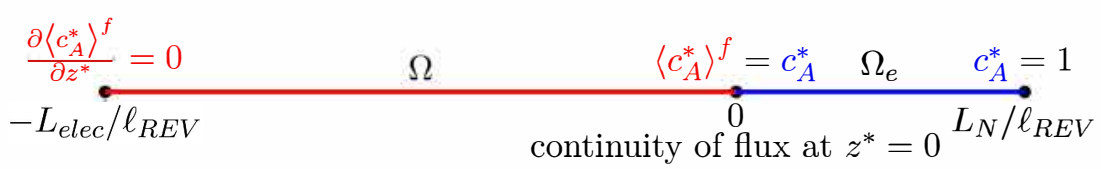

Fig. 5.1. Configuration of the $1 \mathrm{D}$ domain including the electrode $(\Omega)$ and Nernstian layer $\left(\Omega_{e}\right)$.

noted that the reference length $\ell_{R E V}$ is again a choice motivated by the comparison that will be performed with the 3D microscopic model. A more physcically relevant scaling in space would indeed rather be $L_{\text {elec }}$. If, at the underlying pore-scale, periodicity is assumed in the $\mathrm{x}$ and $\mathrm{y}$ directions, as in Section 3, the problem to be solved is now $1 \mathrm{D}$ (in the z-direction) and the configuration is the one represented in Fig. 5.1. The dimensionless form of the closure problem yielding $\mathbf{D}_{\text {eff }}$ is

$$
\begin{aligned}
& \nabla^{* 2} \mathbf{b}^{*}=0 \quad \text { in } \mathrm{V}_{f} \\
& \mathbf{n} \cdot \nabla^{*} \mathbf{b}^{*}=-\mathbf{n} \quad \text { at } \mathrm{A}_{s f} \\
& \left\langle\mathbf{b}^{*}\right\rangle=0 \\
& \mathbf{b}^{*}\left(\mathbf{r}^{*}\right)=\mathbf{b}^{*}\left(\mathbf{r}^{*}+\ell_{i}^{*}\right)
\end{aligned}
$$

with

$\mathbf{D}_{\text {eff }}=\mathcal{D}_{A}\left(\mathbf{I}+\frac{1}{V_{f}^{*}} \int_{\mathrm{A}_{s f}} \mathbf{n} \mathbf{b}^{*} d A^{*}\right)$

Relevant boundary conditions at the dividing surface between a fluid and a porous medium are a critical issue when modeling mass transfer coupled to reaction in a system like the one under consideration here. In the present case, continuity of the concentration and flux expressed in Eqs. (21c) and (21d) at $z^{*}=0$ is adopted and the justification of such a choice is provided in Appendix B.

\subsection{Effective coefficients}

We shall first present results on the effective coefficients (porosity, specific area and effective diffusion coefficient) and examine their dependence upon the pore connection window size for different pore arrangements. As quoted before, in the absence of detailed microstructural experimental investigations, three preliminary isotropic model structures may be considered, namely cubic (C), body-centered cubic (BCC) and face-centered cubic (FCC), the representative unit cells of which are depicted in Fig. 3.1. For these isotropic structures, $\mathbf{D}_{\text {eff }}=\mathcal{D}_{\text {eff }} \mathbf{I}$ which, together with $\varepsilon_{f}$ and $a_{v}^{*}=a_{v} \ell_{R E V}$, depend only on the relative pore connec- tion window size $d_{c} / d_{s}$. The effective diffusion coefficient $\mathcal{D}_{\text {eff }}$ was computed by solving the closure problem in Eqs. (16) (or (22)) on the corresponding periodic unit cell of each structure using COMSOL Multiphysics.

The porosity and dimensionless specific area are represented versus $d_{c} / d_{s}$ in Fig. 5.2 for $0 \leqslant d_{c} / d_{s} \leqslant 20 \%$. As expected, $\varepsilon_{f}$ increases with $d_{c} / d_{s}$ while $a_{v}^{*}$ decreases. The variations of both coefficients remain small over the investigated range of $d_{c} / d_{s}$ as they are less than $7 \%$ for the FCC structure for which porosity and specific area are the largest due to its optimal arrangement.

In Fig. 5.3, we have reported the normalized effective diffusion coefficient $\varepsilon_{f} \mathcal{D}_{\text {eff }} / \mathcal{D}_{A}$ versus $d_{c} / d_{s}$ for the three different types of sphere packing under consideration. For each of them, the effective diffusion coefficient increases almost linearly with the pore connection window size. The FCC structure yields the largest values of $\mathcal{D}_{\text {eff }}$, the simple cubic packing providing the smallest while intermediate values are obtained for the BCC arrangement.

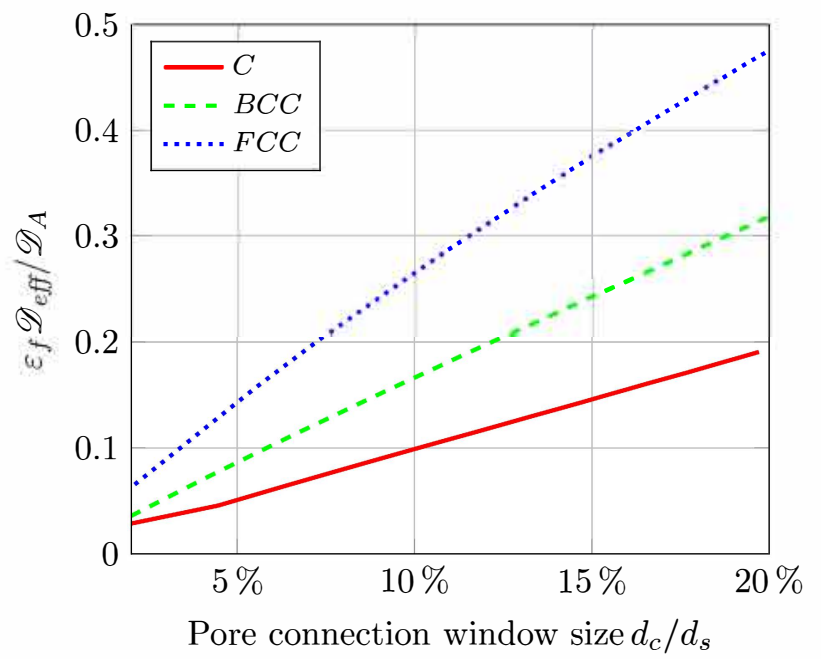

Fig. 5.3. Normalized effective diffusion coefficient $\varepsilon_{f} \mathcal{D}_{\text {eff }} / \mathcal{D}_{A}$ for the three mode structures of Fig. 3.1 versus $d_{c} / d_{s}$.

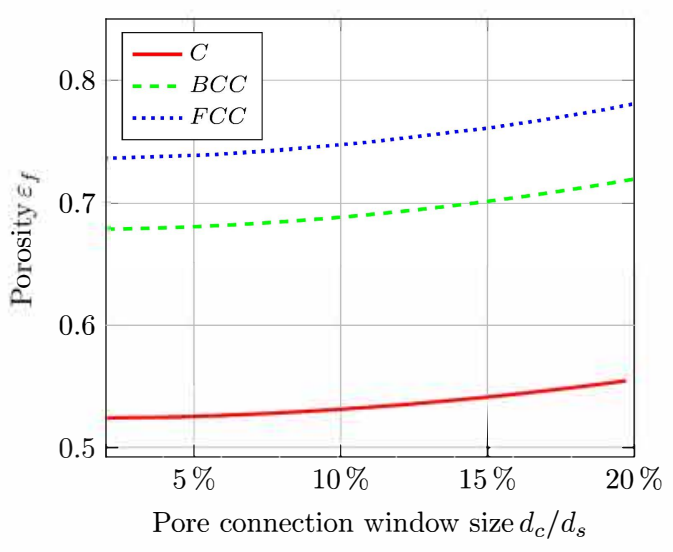

a)

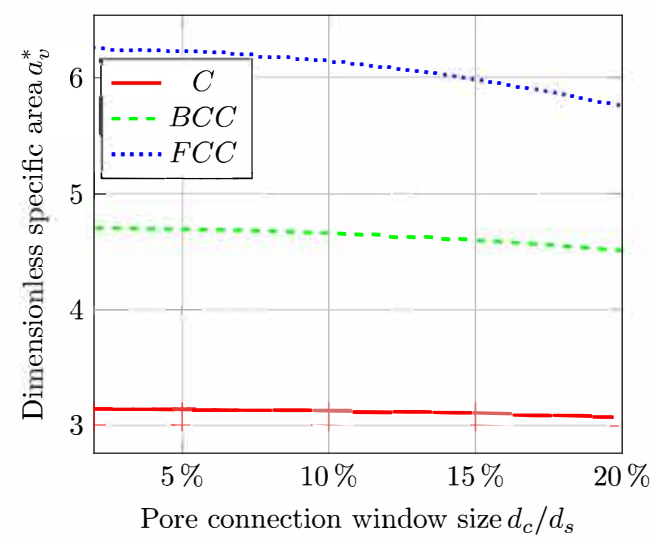

b)

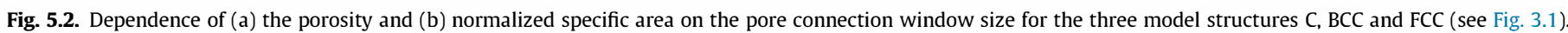


For the results presented in the next two sections, the FCC structure was considered and the problem in Eqs. (21) was solved using COMSOL Multiphysics. Chronoamperometry simulations were first carried out for comparison with DNS and voltammetry simulations were performed in a second step for comparison with experiments. The effect of the electrode thickness $L_{\text {elec }}$, pore microstructure and electron transfer rate constant $k_{0}$ was investigated.

\subsection{Chronoamperometry simulations}

Simulations of chronoamperometry using the 1D-macroscopic effective model (Eqs. (21)) were carried out in the same conditions as for 3D DNS at the microscale presented in Section 3, i.e. using parameters indicated in Table 3.1. These parameters are close to laboratory testing conditions reported in Karajić et al. (2015), Reculusa et al. (2011), Szamocki et al. (2006). For this microstructure, $\varepsilon_{f}=0.763, a_{v}^{*}=5.985$ and $\varepsilon_{f} \mathcal{D}_{\text {eff }} / \mathcal{D}_{A}=0.364$. As in the $3 \mathrm{D}$ case, we took $L_{\text {elec }}^{*}=30$ and $L_{N}^{*} \simeq 12\left(L_{N}=20 \mu \mathrm{m}\right)$. The $1 \mathrm{D}$ simulations were performed with a mesh composed of 700 grid blocks, a spatial discretization that was checked to ensure mesh conver- gence of the solution. Special care was taken to account for the sharp concentration gradient present at $z^{*}=0$ and this was handled by locally refining the mesh on both sides of this interface.

For comparison purposes, the field of $c_{A}^{*}$ obtained from 3D DNS of Eqs. (8) was averaged on each FCC unit cell and then compared with $\left\langle c_{A}^{*}\right\rangle^{f}$, solution of the macroscopic effective model. In Fig. 5.4, dimensionless concentration profiles along the electrode obtained for $k_{0}=10^{-8} \mathrm{~cm} / \mathrm{s}$ are represented for several dimensionless times ranging from 10 to 200 .

Clearly, the agreement between the two approaches is excellent, confirming that the macroscopic model correctly captures the diffusion/reaction mechanisms under consideration. It must be emphasized that the computational resources and time are considerably smaller for simulations of the 1D macroscopic model in comparison to 3D DNS. In the case under study the computational time is only $1 s$ with the 1D upscaled model, leading to a speed-up of roughly 3120 .

To complete the validation, it is important to compare the total intensity available at the electrode estimated with the upscaled model and given by Eq. (20) to that in its original form in the 3D model expressed in Eq. (7). In Fig. 5.5, we have represented the

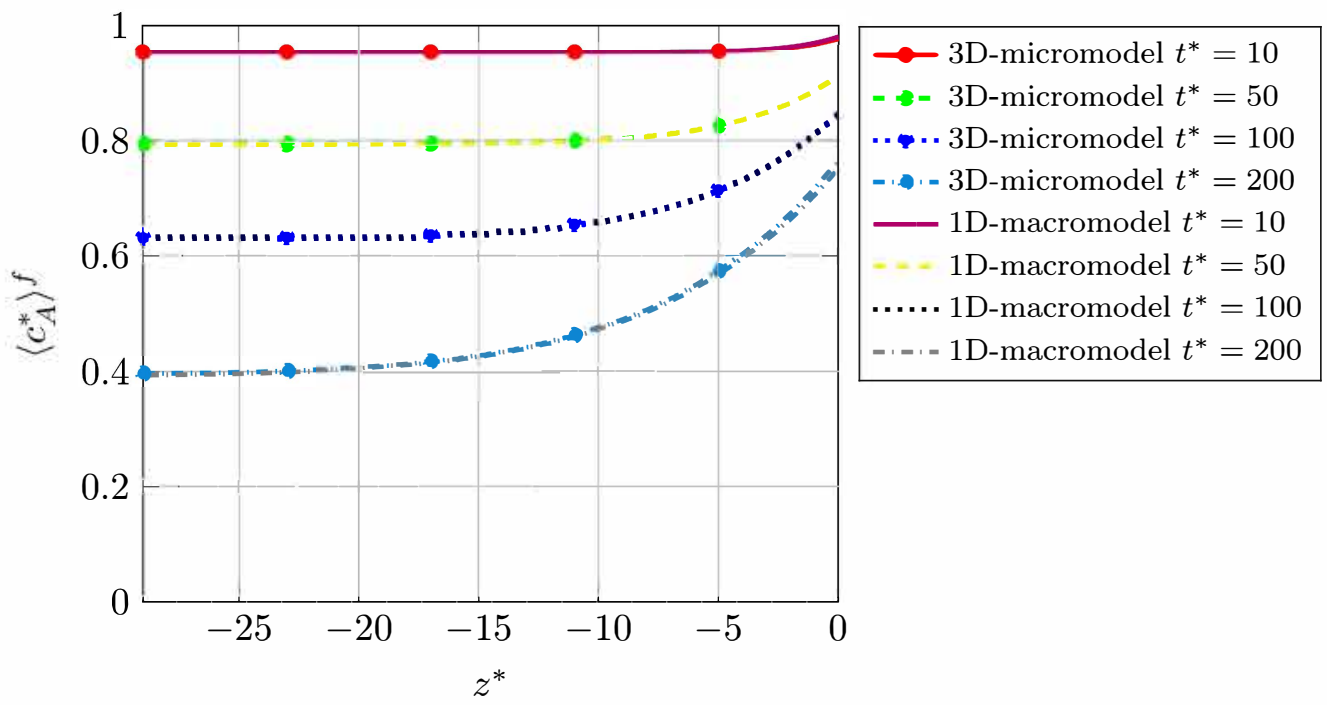

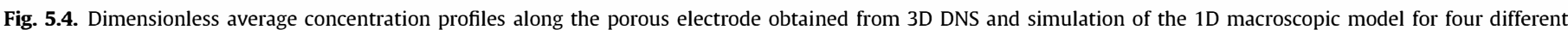
dimensionles times. $k_{0}=10^{-8} \mathrm{~cm} / \mathrm{s}$.

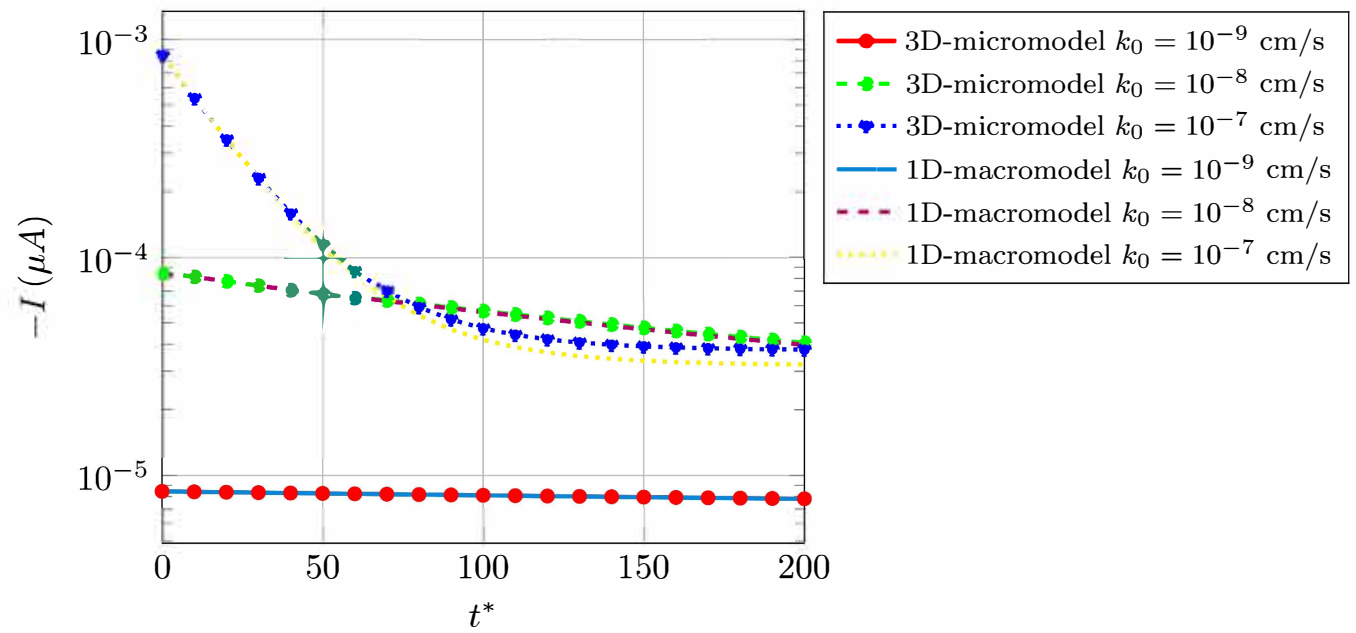

Fig. 5.5. Evolution of the current available at the electrode computed with the 3D microscopic (Eq. (7)) and 1D macroscopic (Eq. (20)) models. 


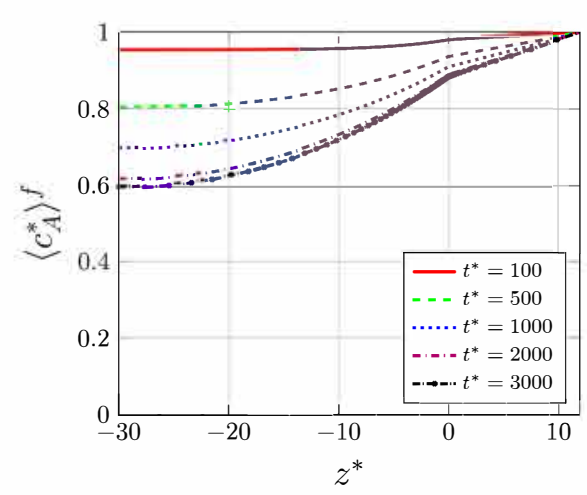

a)

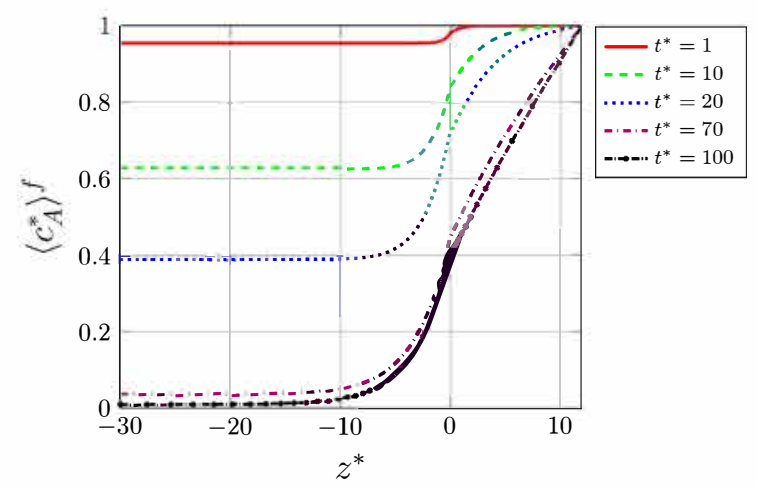

b)

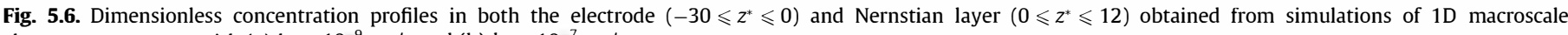
chronoamperometry with (a) $k_{0}=10^{-9} \mathrm{~cm} / \mathrm{s}$ and (b) $k_{0}=10^{7} \mathrm{~cm} / \mathrm{s}$.

evolution of $I$ computed from these two expressions, considering three values of the electron transfer rate constant $k_{0}=10^{-9} \mathrm{~cm} / \mathrm{s}$, $k_{0}=10^{-8} \mathrm{~cm} / \mathrm{s}$ and $k_{0}=10^{-7} \mathrm{~cm} / \mathrm{s}$. Again, the agreement in each case is excellent confirming the accuracy of the macroscopic model. The excellent agreement is consistent with the value of the kinetic number which, for $k_{0}=10^{-7} \mathrm{~cm} / \mathrm{s}$, is $K i=\frac{k_{0} \alpha_{A} d_{s}}{\mathcal{D}_{A}} \simeq 0.0046$ for the case under consideration here, a value satisfying the constraint (A.8) on which the estimation in Eq. (20) relies.

Evolutions of the concentration profiles within the electrode $\left\langle c_{A}^{*}\right\rangle^{f}$ and the Nernstian layer $c_{A}^{*}$ obtained from the simulation of chronoamperometry of the 1D macroscopic model are depicted in Fig. 5.6 for $k_{0}=10^{-9} \mathrm{~cm} / \mathrm{s}$ and $k_{0}=10^{-7} \mathrm{~cm} / \mathrm{s}$, showing the concentration decrease with time due to diffusion and reaction in the electrode and diffusion from the Nernstian layer to the porous region. In particular, when $k_{0}$ is large, almost no concentration gradient is present in the electrode as the mechanism is strongly dominated by the electrochemical reaction process, the entire electrode operating in a mass transfer limited regime. Conversely, when $k_{0}$ is small (see Fig. 5.6a for $k_{0}=10^{-9} \mathrm{~cm} / \mathrm{s}$ ) diffusion is significant and a concentration gradient persists in the electrode domain corresponding to an intermediate regime. A stationary regime is also observed when the balance between diffusion and reaction is reached on the overall system. The equilibrium characteristic time strongly decreases when $k_{0}$ increases. Indeed, steady state is reached at $t^{*} \simeq 3000$ for $k_{0}=10^{-9} \mathrm{~cm} / \mathrm{s}$ and $t^{*} \simeq 100$ for $k_{0}=10^{-7} \mathrm{~cm} / \mathrm{s}$.

The current density averaged on the entire electrode $\frac{1}{|\Omega|} \int_{\Omega}\langle j\rangle_{s f} \mathrm{~d} V=\frac{I}{\left|I_{s f}\right|}$ was also computed according to the relationship in Eq. (18) and its evolution is represented in Fig. 5.7 for three values of $k_{0}$, namely $k_{0}=10^{-9} \mathrm{~cm} / \mathrm{s}, k_{0}=10^{-8} \mathrm{~cm} / \mathrm{s}$ and $k_{0}=10^{-7} \mathrm{~cm} /$ s. For small values of $k_{0}$ (i.e. $k_{0}=10^{-9} \mathrm{~cm} / \mathrm{s}$ ), the average current density produced by the electrode is small and remains quasi constant over time. For larger values of $k_{0}$, a large current is produced at the early stage of the process and strongly decreases to reach a plateau at equilibrium.

The influence of the microstructures on the electrical production is illustrated in Fig. 5.8. Keeping all parameters the same, the current intensity per unit volume of the electrode $\frac{1}{|\Omega|} \int_{\Omega} i_{v} \mathrm{~d} V=\frac{1}{|\Omega|}$ was computed for the three different pore structures C, BCC and FCC and two values of the electron transfer rate constant $k_{0}=10^{-7} \mathrm{~cm} / \mathrm{s}$ and $k_{0}=10^{-9} \mathrm{~cm} / \mathrm{s}$. For both values of $k_{0}$, the largest value of the current intensity is produced on the

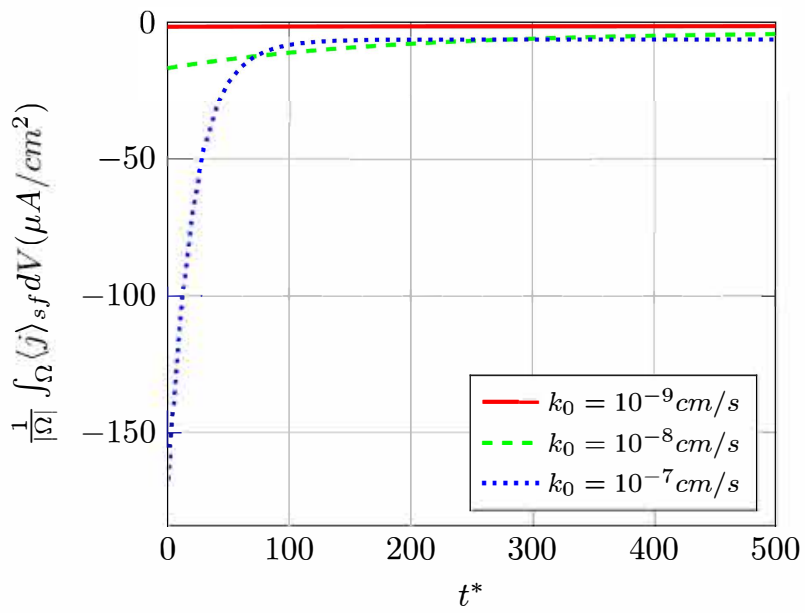

Fig. 5.7. Evolution of the average current density on the electrode for three values of $k_{0}$.

FCC structure, while the lowest is obtained on the $C$ structure. This can be explained by the values of $\varepsilon_{f}, a_{v}$ and $\mathcal{D}_{\text {eff }}$ that are the largest for the FCC arrangement. For all three structures, the current intensity decreases with time and reaches a plateau at equilibrium that is attained with almost the same characteristic time for the three structures under investigation. The amplitude of variation of the current intensity over time decreases strongly when $k_{0}$ increases and the rate of decrease is much stronger for a large value of $k_{0}$.

\subsection{Voltammetry - comparison with experimental data}

The objective in this section is to compare our simulation of the 1D upscaled model with experimental data reported in Reculusa et al. (2011) in which the intensity of porous gold coated electrodes of different thicknesses was recorded during voltammetry. The porous electrode thicknesses correspond to 7,27 and 80 halflayers of silica templates made of beads of $1.2 \mu \mathrm{m}$ in diameter and deposited onto a gold wire of $50 \mu \mathrm{m}$. Voltammetry was run under a potential scan from $E=0.2$ to $-0.4 \mathrm{~V}$ at the same rate $r_{E}=5 \mathrm{mV} / \mathrm{s}$. The electrode was immersed in a $0.05 \mathrm{M} \mathrm{H}_{2} \mathrm{SO}_{4}$ aqueous solution saturated with oxygen. The number of electron transferred in that case corresponds to $n=2$.

The diffusion coefficient of oxygen in a $0.05 \mathrm{M} \mathrm{H}_{2} \mathrm{SO}_{4}$ solution can be taken as $\mathcal{D}_{A}=2.10^{-9} \mathrm{~m}^{2} / \mathrm{s}$ (see Bala, 1984) while Nernstian 


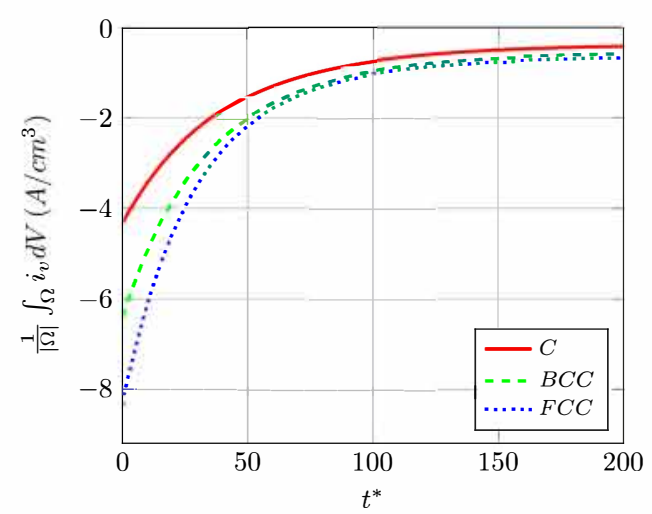

a)

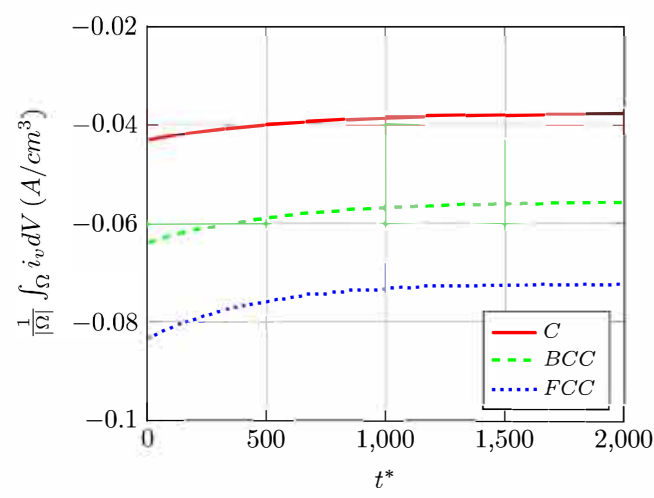

b)

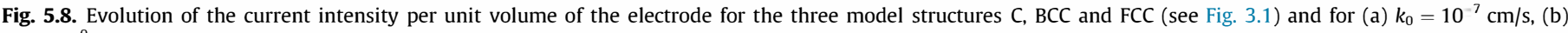
$k_{0}=10^{-9} \mathrm{~cm} / \mathrm{s}$.

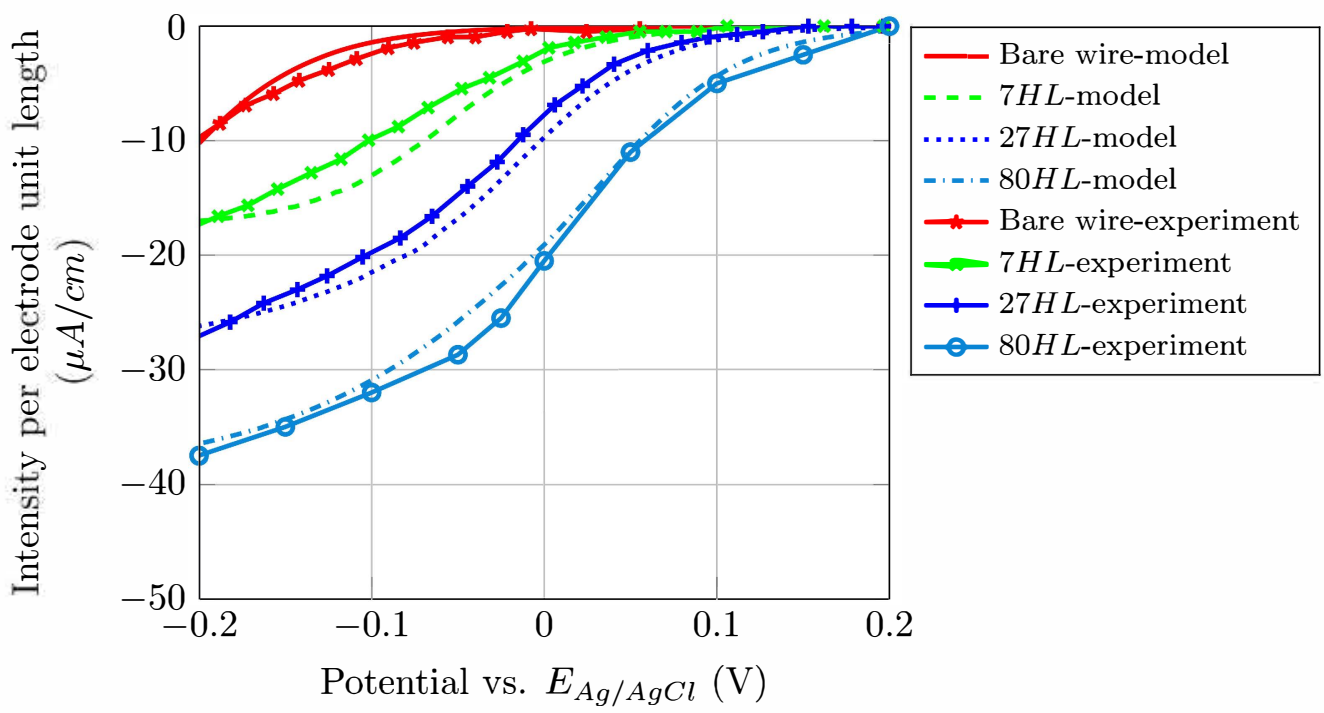

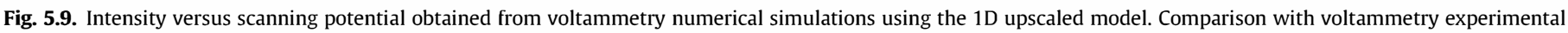
data reported in Reculusa et al. (2011).

layer thicknesses $L_{N}=65 \mu \mathrm{m}$ for bare wire, 27 and 80 half-layers and $L_{N}=110 \mu \mathrm{m}$ for 7 half-layers were adopted. It must be noted that the Nernstian layer thickness should be considered as a timedependent parameter (Molina et al., 2013). For the sake of simplicity, this dependence was disregarded as a first approach in the present work. Two other coefficients need to be chosen, namely the electron transfer rate constant, $k_{0}$, and the charge transfer coefficient, $\alpha$, for which no values are available so far. Depending on the material coating the pores and the texture of the surface coating, $k_{0}$ can vary over several orders of magnitude (Jirkovský et al., 2010; Wang et al., 2013). Again, the value of $k_{0}$ may be very small for a redox reaction without catalysis (Renslow et al., 2011). As a consequence the values of $k_{0}$ and $\alpha$ were adjusted to achieve the best fit with the intensity curve corresponding to the 27 halflayers electrode thickness of Fig. 5.2 in Reculusa et al. (2011). The values $k_{0}=10^{-9} \mathrm{~cm} / \mathrm{s}$ and $\alpha=0.66$ for a gold electrode yielded the best fit. They were kept for all the simulations performed with the other electrode thicknesses ( 7 and 80 half-layers). For the whole range of the scanning potential investigated in the simulation of voltammetry, the value of the kinetic number $K i=\frac{k_{0} \alpha_{A} d_{S}}{\mathcal{D}_{A}}$

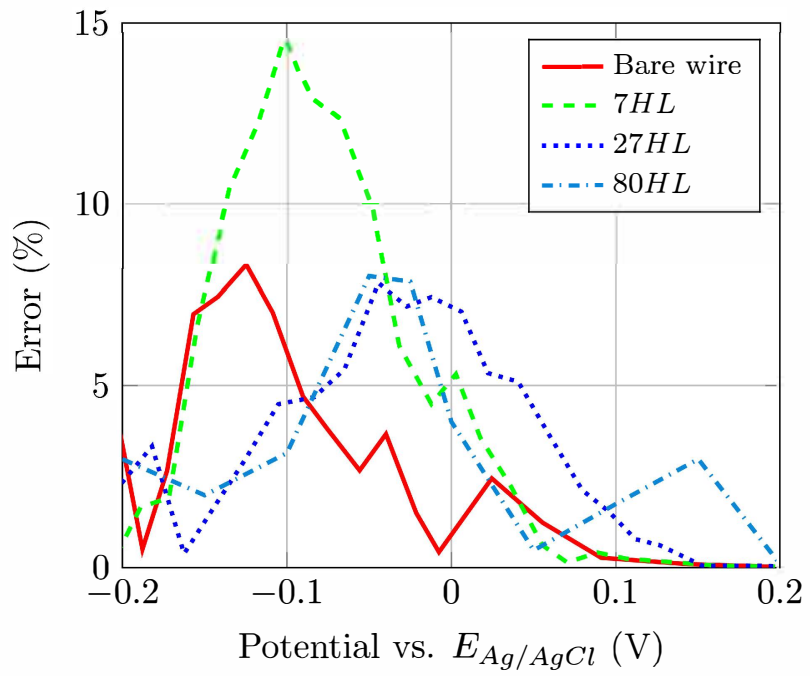

Fig. 5.10. Relative error between numerical results and experimental data. 
remains between $3.410^{-6}$ and 0.09 and this ensures that the estimation of the current intensity using Eq. (20) is valid.

In Fig. 5.9, we have represented the intensity per electrode unit length versus the scanning potential obtained from our numerical simulation together with the experimental results of Fig. 5.2 in Reculusa et al. (2011). The agreement of our simulation results with measurements on the electrodes made of 7 and 80 halflayers is quite satisfactory. The case of the bare-wire is quite different from the others as no porous medium is present and the gold layer may lead to a different value of $\alpha$. The simulation in that case was carried out using Eq. (8a) in the Nerrnstian diffusion layer with the boundary conditions B.C. 1 in Eq. (8b) applied at $z^{*}=0$ and B. C.3 in Eq. (8d) together with initial condition in Eq. (8e) for $0<z^{*}<L_{N}^{*}$. Indeed, a very good fit was obtained by taking $\alpha=0.61$ in that case as reported in Fig. 5.9. It must be observed that, for a given potential, the absolute intensity increases with the porous electrode thickness. To better evaluate the fit, the relative error between the numerical results and experimental data on the current per unit length of the electrode as represented in Fig. 5.9 are reported in Fig. 5.10. The reference value of the current was taken as its maximum experimental value for each electrode, i.e. at a potential of $-0.2 \mathrm{~V}$. The maximum error is about $14 \%$ for the 7 half-layers electrode at a potential of $-0.1 \mathrm{~V}$ while the error is around $5 \%$ on average for the whole dataset.

\section{Conclusions}

A multiscale approach to model porous electrodes used in active or passive electro-analytical devices was developed in this work in the context of reduction reaction of oxygen at the cathode in the absence of catalysis. Within this context, the pore-scale model consists in a coupled diffusion-electrochemical reaction which can be solved using DNS at the cost of very significant computational resources. With the aim of developing a strategy to analyze the relationships between the porous medium microstructure and the macroscopic properties(i.e. the current intensity available at the electrode), an upscaled model operating at the scale of an effective equivalent medium was derived by upscaling the porescale model with the aid of the volume averaging method. The effective diffusion tensor present in this model can be simply determined from the solution of an intrinsic closure problem on a representative elementary volume of the microsctructure. This upscaled model contains all the essential information from the pore-scale and is far less computationally demanding. Its validity and accuracy was proven through comparative simulations of amperometry at both the pore-scale and macroscale showing an excellent agreement between the two approaches on the concentration profiles and macroscopic current intensity available at the electrode. The speed-up of the computational procedure achieved by using the effective macroscopic model is roughly 3100 compared to DNS. Various porous model structures (C, BCC, FCC) and values of the parameters (electron transfer rate constant) were investigated as illustrative examples.

Simulation of voltammetry tests performed with the upscaled model were compared to intensity versus scanning potential experimental data reported in the literature. Parameters were chosen as close as possible to the corresponding experimental configuration except the Nernstian layer thickness and the electron transfer coefficient that are unknown in the experimental approach and need to be adjusted. A very good agreement was achieved between predictions from the upscaled model and the experimental results confirming the relevance of this model.

Further work is necessary to complete the modeling of the electrochemical process beyond the simple case of oxygen reduction in order to analyze more complex situations involving enzymes as a catalyst in the case of Direct Electron Transfer or Mediated Electron Transfer for which a mediator species shuttles electrons between enzymes' active centers and the electrode internal fluid-solid interfaces.

The high potential capability of the multiscale approach developed in the present work opens the way to investigate the optimal design of the porous architecture in order to improve the electrode effectiveness and this represents a key step to progress in the development of these innovative micro-electrochemical devices that needs to be addressed from the point of view of chemical engineering.

\section{Acknowledgement}

This work was supported by the LabEx AMADEus (ANR-10LABX-42) within IdEx Bordeaux (ANR-10-IDEX-03-02), i.e. the "Investissements d'Avenir Programme" of the French government managed by the Agence Nationale de la Recherche.

\section{Appendix A. Volume averaging and derivation of the upscaled model}

In this Appendix, the upscaling procedure to derive the macroscopic diffusion/reaction equation by volume averaging the microscopic IBVP is reported. Volume averaging is applied according to the following four main steps. More complete details can be found in Chap. 1 of Whitaker (1999) (see also Valdés-Parada et al., 2011). Again, we use $c_{A}$ to denote $c_{A}(\mathbf{r}, t)$.

\section{A.1. Step 1: Application of the superficial average operator}

The superficial average operator is applied to the microscale IBVP on $c_{A}$ and, with the purpose of deriving a model involving $\left\langle c_{A}\right\rangle$ (or $\left\langle c_{A}\right\rangle^{f}$ ) only, time and spatial derivation must be interchanged with volume averaging. This is achieved by using the general transport theorem, which, since the averaging volume is fixed in time and the porous medium is rigid, reduces to

$\left\langle\frac{\partial c_{A}}{\partial t}\right\rangle=\frac{\partial\left\langle c_{A}\right\rangle}{\partial t}$

and the spatial averaging theorem (or Leibniz rule) given by Truesdell and Toupin (1960), Whitaker (1999)

$\left\langle\nabla c_{A}\right\rangle=\nabla\left\langle c_{A}\right\rangle+\frac{1}{V} \int_{\mathrm{A}_{s f}} \mathbf{n} c_{A} d A$

together with a straightforward similar form for the divergence operator. When this theorem is employed twice and by making use of the boundary condition at $A_{s f}$ (see Eq. (8b)), one arrives at the following average form

$$
\begin{aligned}
\varepsilon_{f} \frac{\partial\left\langle c_{A}\right\rangle^{f}}{\partial t}= & \nabla \cdot\left[\mathcal{D}_{A}\left(\varepsilon_{f} \nabla\left\langle c_{A}\right\rangle^{f}+\left\langle c_{A}\right\rangle^{f} \nabla \varepsilon_{f}+\frac{1}{V} \int_{A_{s f}} \mathbf{n} c_{A} \mathrm{~d} A\right)\right] \\
& -k_{0} \alpha_{A} a_{v}\left\langle c_{A}\right\rangle_{s f}
\end{aligned}
$$

where the intrinsic average concentration was used instead of the superficial average.

\section{A.2. Step 2: Decomposition of $c_{A}$ and simplifications due to length-scale constraints}

The average Eq. (A.3) contains both average and point-wise concentrations. To remove the latter, the spatial decomposition

$c_{A}=\left\langle c_{A}\right\rangle^{f}+\tilde{c}_{A}$ 
is introduced (Gray, 1975), where $\tilde{c}_{A}$ is the deviation of concentration which fluctuates at a typical length-scale $\ell_{p}$ while $\left\langle c_{A}\right\rangle^{f}$ experiences significant variations at the scale $L$. It should be noted that a consequence of this decomposition is $\left\langle\tilde{c}_{A}\right\rangle=0$. When this decomposition is inserted into Eq. (A.3), one gets

$$
\begin{aligned}
\varepsilon_{f} \frac{\partial\left\langle c_{A}\right\rangle^{f}}{\partial t}= & \nabla \cdot\left[\mathcal { D } _ { A } \left(\varepsilon_{f} \nabla\left\langle c_{A}\right\rangle^{f}+\left\langle c_{A}\right\rangle^{f} \nabla \varepsilon_{f}+\frac{1}{V} \int_{A_{s f}} \mathbf{n}\left\langle c_{A}\right\rangle^{f} \mathrm{~d} A\right.\right. \\
& \left.\left.+\frac{1}{V} \int_{\mathrm{A}_{s f}} \mathbf{n} \tilde{c}_{A} \mathrm{~d} A\right)\right]-k_{0} \alpha_{A} a_{v}\left\langle c_{A}\right\rangle_{s f}
\end{aligned}
$$

Let us now focus attention onto the area integral term $\frac{1}{V} \int_{A_{s f}} \mathbf{n}\left\langle c_{A}\right\rangle^{f} \mathrm{~d} A$ on the right hand side of Eq. (A.5). This term must be evaluated at the centroid $\mathbf{x}$ of the averaging volume $V$ and requires first the evaluation of $\left\langle c_{A}\right\rangle^{f}$ at any point $\mathbf{x}+\mathbf{y}$ on $\mathrm{A}_{s f}$ contained in $\mathrm{V}$, making this term non-local. A Taylor expansion given by

$$
\left.\left\langle c_{A}\right\rangle^{f}\right|_{\mathbf{x}+\mathbf{y}}=\left.\left\langle c_{A}\right\rangle^{f}\right|_{\mathbf{x}}+\left.\mathbf{y} \cdot \nabla\left\langle c_{A}\right\rangle^{f}\right|_{\mathbf{x}}+\frac{1}{2} \mathbf{y y}:\left.\nabla \nabla\left\langle c_{A}\right\rangle^{f}\right|_{\mathbf{x}}+\ldots
$$

may now be employed and, when introduced back into Eq. (A.5) together with the orders of magnitude estimates to identify the significant terms, it is not hard to deduce that Eq. (A.5) can be simplified to (see Whitaker, 1999 for more details)

$$
\begin{aligned}
\varepsilon_{f} \frac{\partial\left\langle c_{A}\right\rangle^{f}}{\partial t}= & \nabla \cdot\left[\mathcal{D}_{A}\left(\varepsilon_{f} \nabla\left\langle c_{A}\right\rangle^{f}+\frac{1}{V} \int_{\mathrm{A}_{s f}} \mathbf{n} \tilde{c}_{A} \mathrm{~d} A\right)\right] \\
& -k_{0} \alpha_{A} a_{v}\left(\left\langle\tilde{c}_{A}\right\rangle_{s f}+\left\langle\left\langle c_{A}\right\rangle^{f}\right\rangle_{s f}\right)
\end{aligned}
$$

where we have introduced the decomposition of the concentration into the reactive term. In addition, it can be shown that, whenever the pore-scale kinetic number $K i=\frac{k_{0} \alpha_{A} \ell_{p}}{\mathcal{D}_{A}}$ satisfies

$K i \ll 1$

we have $\tilde{c}_{A} \ll\left\langle c_{A}\right\rangle^{f}$ at $\mathrm{A}_{\mathrm{sf}}$ (see Whitaker, 1999). Under these circumstances, Eq. (A.7) can be simplified to the following form

$\varepsilon_{f} \frac{\partial\left\langle c_{A}\right\rangle^{f}}{\partial t}=\nabla \cdot\left[\mathcal{D}_{A}\left(\varepsilon_{f} \nabla\left\langle c_{A}\right\rangle^{f}+\frac{1}{V} \int_{\mathrm{A}_{s f}} \mathbf{n} \tilde{c}_{A} \mathrm{~d} A\right)\right]-k_{0} \alpha_{A} a_{v}\left\langle\left\langle c_{A}\right\rangle^{f}\right\rangle_{s f}$

A close attention needs to be dedicated to the last area integral term on the right hand side of this equation since it is non-local. Indeed, when the Taylor expansion of Eq. (A.6) is introduced in this integral, one has

$$
\left\langle\left\langle c_{A}\right\rangle^{f}\right\rangle_{s f}=\left\langle c_{A}\right\rangle^{f}+\langle\mathbf{y}\rangle_{s f} \cdot \nabla\left\langle c_{A}\right\rangle^{f}+\frac{1}{2}\langle\mathbf{y y}\rangle_{s f}: \nabla \nabla\left\langle c_{A}\right\rangle^{f}+\ldots
$$

By estimating the orders of magnitude of the integral terms, it can be proven that $\left\langle\left\langle c_{A}\right\rangle^{f}\right\rangle_{s f} \approx\left\langle c_{A}\right\rangle^{f}$ (Whitaker, 1999). As a consequence the average Eq. (A.9) can be simplified to the following form

$$
\varepsilon_{f} \frac{\partial\left\langle c_{A}\right\rangle^{f}}{\partial t}=\nabla \cdot\left[\mathcal{D}_{A}\left(\varepsilon_{f} \nabla\left\langle c_{A}\right\rangle^{f}+\frac{1}{V} \int_{A_{s f}} \mathbf{n} \tilde{c}_{A} \mathrm{~d} A\right)\right]-k_{0} \alpha_{A} a_{v}\left\langle c_{A}\right\rangle^{f}
$$

The boundary condition at the solid-fluid interface can be written as

$$
-\mathbf{n} \cdot \mathcal{D}_{A} \nabla \tilde{c}_{A}=\mathbf{n} \cdot \mathcal{D}_{A} \nabla\left\langle c_{A}\right\rangle^{f}+k_{0} \alpha_{A}\left\langle c_{A}\right\rangle^{f} \quad \text { at } A_{s f}
$$

At this stage, the model remains unclosed since $\tilde{c}_{A}$ is still present in the averaged mass balance equation of species $A$.

\section{A.3. Step 3: Closure}

The idea is now to form an IBVP on $\tilde{c}_{A}$ which solution can be inserted into the averaged equation. This can be achieved by subtracting Eq. (A.11) divided by $\varepsilon_{f}$ from the initial pore-scale Eq. (2a) and by using the boundary condition under the form of Eq. (A.12). When the orders of magnitude estimates are employed and the process is considered at a time scale constrained by

$\frac{\mathcal{D}_{A} t}{\ell_{p}^{2}} \gg 1$

it is not hard to deduce that the closure problem can be simplified to

$$
\begin{aligned}
& \nabla^{2} \tilde{c}_{A}=-\underbrace{\frac{\varepsilon_{f}^{-1} k_{0} \alpha_{A} a_{v}}{\mathcal{D}_{A}}\left\langle c_{A}\right\rangle^{f}}_{\text {reactive volume source }} \text { in } \mathrm{v}_{f} \\
& -\mathbf{n} \cdot \mathcal{D}_{A} \nabla \tilde{c}_{A}=\underbrace{\mathbf{n} \cdot \mathcal{D}_{A} \nabla\left\langle c_{A}\right\rangle^{f}}_{\text {diffusive surface source }}+\underbrace{k_{0} \alpha_{A}\left\langle c_{A}\right\rangle^{f}}_{\text {reactive surface source }} \\
& \tilde{c}_{A}(\mathbf{r})=\tilde{c}_{A}\left(\mathbf{r}+\ell_{i}\right)
\end{aligned}
$$

It should be noted that the problem is solved on a REV that is periodic with a period $\ell_{i}$ in the $i$ th-direction so that the external boundary condition is replaced by the periodic condition in (A.14c).

This problem has a linear structure, so the solution on $\tilde{c}_{A}$ can be sought in terms of a linear combination of the sources identified in Eqs. (A.14a) and (A.14b) under the form

$\tilde{c}_{A}=\mathbf{b} \cdot \nabla\left\langle c_{A}\right\rangle^{f}+s\left\langle c_{A}\right\rangle^{f}$

b and $s$ being the closure variables which can be chosen to obey the following intrinsic boundary value problems (Whitaker, 1999)

$$
\begin{aligned}
& \nabla^{2} \mathbf{b}=0 \quad \text { in } \vee_{f} \\
& \mathbf{n} \cdot \nabla \mathbf{b}=-\mathbf{n} \quad \text { at } A_{s f} \\
& \langle\mathbf{b}\rangle=0 \\
& \mathbf{b}(\mathbf{r})=\mathbf{b}\left(\mathbf{r}+\ell_{i}\right)
\end{aligned}
$$

and

$$
\begin{aligned}
& \nabla^{2} s=-\frac{\varepsilon_{f}^{-1} k_{0} \alpha_{A} a_{v}}{\mathcal{D}_{A}} \quad \text { in } \mathrm{V}_{f} \\
& \mathbf{n} \cdot \nabla s=-\frac{k_{0} \alpha_{A}}{\mathcal{D}_{A}} \quad \text { at } \mathrm{A}_{s f} \\
& \langle s\rangle=0 \\
& s(\mathbf{r})=s\left(\mathbf{r}+\ell_{i}\right)
\end{aligned}
$$

Note that the conditions in Eqs. (A.16c) and (A.17c) result from the fact that $\left\langle\tilde{c}_{A}\right\rangle=0$ and are required for both closure problems to have a unique solution.

\section{A.4. Step 4: Macroscopic model}

When the representation of $\tilde{c}_{A}$ is reported back into the average Eq. (A.11), one finally obtains the following closed macroscopic equation

$$
\varepsilon_{f} \frac{\partial\left\langle c_{A}\right\rangle^{f}}{\partial t}=\nabla \cdot\left(\varepsilon_{f} \mathbf{D}_{e f f} \cdot \nabla\left\langle c_{A}\right\rangle^{f}\right)+\nabla \cdot\left(\mathbf{u}\left\langle c_{A}\right\rangle^{f}\right)-k_{0} \alpha_{A} a_{v}\left\langle c_{A}\right\rangle^{f}
$$

with the effective parameters $\mathbf{D}_{\text {eff }}$ and $\mathbf{u}$ given by

$$
\begin{aligned}
\mathbf{D}_{\text {eff }} & =\mathcal{D}_{A}\left(\mathbf{I}+\frac{1}{V_{f}} \int_{\mathrm{A}_{s f}} \mathbf{n b} d A\right) \\
\mathbf{u} & =\frac{\mathcal{D}_{A}}{V}\left(\int_{\mathrm{A}_{s f}} \mathbf{n} s d A\right)
\end{aligned}
$$


where $\mathbf{b}$ and $s$ are solution of the closure problems in Eqs. (A.16) and (A.17) respectively. Moreover, from Eq. (A.17b), the order of magnitude estimate for $s$ is $s=\mathbf{O}\left(k_{0} \alpha_{A} \ell_{p} / \mathcal{D}_{A}\right)$ and from Eq. (A.20), one gets $\mathbf{u}=\mathbf{O}\left(k_{0} \alpha_{A}\right)$. This leads to an order of magnitude estimate for the second term on the right hand side of Eq. (A.18) given by $\boldsymbol{\nabla} \cdot\left(\mathbf{u}\left\langle c_{A}\right\rangle^{f}\right)=\mathbf{O}\left(\frac{k_{0} \alpha_{A}}{L}\left\langle c_{A}\right\rangle^{f}\right)$ while the order of magnitude estimate of the macroscopic reactive term is $k_{0} \alpha_{A} a_{v}\left\langle c_{A}\right\rangle^{f}=\mathbf{O}\left(\frac{k_{0} \alpha_{A}}{\ell_{p}}\left\langle c_{A}\right\rangle^{f}\right)$ with the idea that $a_{v}=\mathbf{O}\left(\ell_{p}^{-1}\right)$. This indicates that the macroscopic diffusion/reaction equation may be written as

$\varepsilon_{f} \frac{\partial\left\langle c_{A}\right\rangle^{f}}{\partial t}=\nabla \cdot\left(\varepsilon_{f} \mathbf{D}_{e f f} \cdot \nabla\left\langle c_{A}\right\rangle^{f}\right)-k_{0} \alpha_{A} a_{v}\left\langle c_{A}\right\rangle^{f}$

\section{Appendix B. Boundary condtions at the dividing surface between the electrode and the fluid}

This appendix is dedicated to a justification of the relevance of boundary conditions in Eqs. (21c) and (21d) indicating that both the concentration and flux can be considered as continuous at the dividing surface between the electrode and the fluid. For the sake of simplicity, the discussion is carried out on the dimensional version of the boundary conditions.

A formal derivation of physically justified boundary conditions for mass transfer coupled to reaction between a porous medium and a fluid was proposed in the literature (Valdés-Parada et al., 2006) leading to a jump of the flux at the dividing surface given by

$-\mathbf{n} \cdot \mathcal{D}_{A} \nabla c_{A}+\mathbf{n} \cdot \varepsilon_{f} \mathbf{D}_{\text {eff }} \cdot \nabla\left\langle c_{A}\right\rangle^{f}=K_{\text {eff }}\left\langle c_{A}\right\rangle^{f} \quad$ at $\mathcal{A}_{\text {int }}$

together with a zero jump of the concentration, just as considered in Eq. (21c). The derivation was carried out with the assumption $K i \ll 1$. In Eq. (B.1), $\mathcal{A}_{\text {int }}$ denotes the dividing surface having an area $A_{\text {int }}$ and $K_{\text {eff }}$ is an effective reaction rate coefficient obtained from the solution of a closure problem. Whenever $K i \ll 1$, it can be easily inferred from this closure problem that

$K_{\text {eff }} \simeq \frac{1}{A_{\text {int }}} \int_{\mathcal{A}_{\text {int }}} k_{0} \alpha_{A} d A$

This last approximation was confirmed by numerical results obtained on some model porous structures (Valdés-Parada et al., 2006). This leads to write

$K_{\text {eff }} \simeq k_{0} \alpha_{A}\left(1-\varepsilon_{s}\right)$

where $\varepsilon_{s}$ designates the surface porosity at $\mathcal{A}_{\text {int }}$, i.e. the ratio of the area of the region occupied by the fluid phase within $\mathcal{A}_{\text {int }}$ to $A_{\text {int }}$.

A comparative order of magnitude estimate shall now be carried out between the second term on the left hand side of Eq. (B.1) and $K_{\text {eff }}\left\langle c_{A}\right\rangle^{f}$. The former can be estimated as

$\mathbf{n} \cdot \varepsilon_{f} \mathbf{D}_{\text {eff }} \cdot \nabla\left\langle c_{A}\right\rangle^{f}=\mathbf{0}\left(\varepsilon_{f} \mathcal{D}_{A} \frac{\left\langle c_{A}\right\rangle^{f}}{h}\right)$

$h$ being the size of the portion of the unit cell within the porous medium that is used to compute $K_{\text {eff }}$. Typically, $h=10 \ell_{\text {REV }}$ is necessary to obtain a representative unit cell (see Valdés-Parada et al., 2006) so that

$\mathbf{n} \cdot \varepsilon_{f} \mathbf{D}_{e f f} \cdot \nabla\left\langle c_{A}\right\rangle^{f}=\mathbf{O}\left(\varepsilon_{f} \mathcal{D}_{A} \frac{\left\langle c_{A}\right\rangle^{f}}{10 \ell_{R E V}}\right)$

Consequently, when $K i$ is such that

$K i \ll \frac{\ell_{p}}{10 \ell_{R E V}} \frac{\varepsilon_{f}}{1-\varepsilon_{s}}$
$K_{\text {eff }}\left\langle c_{A}\right\rangle^{f} \ll \mathbf{n} \cdot \varepsilon_{f} \mathbf{D}_{\text {eff }} \cdot \nabla\left\langle c_{A}\right\rangle^{f}$ and the boundary condition in Eq. (B.1) can be simplified to a zero jump of the flux at the dividing surface, i.e.

$\mathbf{n} \cdot \mathcal{D}_{A} \nabla c_{A}=\mathbf{n} \cdot \varepsilon_{f} \mathbf{D}_{\text {eff }} \cdot \nabla\left\langle c_{A}\right\rangle^{f} \quad$ at $\mathcal{A}_{\text {int }}$

which corresponds to the condition used in the statement of the macroscopic dimensionless problem in Eqs. (21).

To be more specific, a quantitative analysis can be made taking the particular FCC structure under consideration that best represents the real configuration. For this structure, $\varepsilon_{s}$ takes the expression

$\varepsilon_{s}=\left[\pi / 4-\cos ^{-1}\left(\sqrt{1-\left(\frac{d_{c}}{d_{s}}\right)^{2}}\right)\right] \frac{1}{1-\left(\frac{d_{c}}{d_{s}}\right)^{2}}+\frac{d_{c}}{d_{s}} \frac{1}{\sqrt{1-\left(\frac{d_{c}}{d_{s}}\right)^{2}}}$

while $\ell_{R E V}=\sqrt{2} d_{s} \sqrt{1-\left(\frac{d_{c}}{d_{s}}\right)^{2}}$. Taking $\ell_{p}=d_{s}$ in (B.6), it can be concluded that the continuity of the flux at the dividing surface expressed in Eq. (21c) remains valid provided

$K i \ll \frac{1}{10 \sqrt{2}} \frac{\varepsilon_{f}}{1-\varepsilon_{s}} \frac{1}{\sqrt{1-\left(\frac{d_{c}}{d_{s}}\right)^{2}}}$

It can be easily shown that the right hand side of (B.9) is minimum when $\frac{d_{c}}{d_{s}}=0$ (i.e. in the limiting case of touching spheres) yielding $K i \ll 0.24$ as a safe constraint. Clearly, for all cases under consideration in the present work, this constraint is satisfied, justifying to employ continuity of the flux (and of the concentration) as boundary conditions between the porous electrode and the fluid.

\section{References}

Bala, H., 1984. Limiting diffusional currents of oxygen reduction in $0.5-11 \mathrm{~m}$ sulphuric acid. Zeitschrift Phys. Chem. Neue Folge, 91-129.

Barnes, E.O., Chen, X., Li, P., Compton, R.G., 2014. Voltammetry at porous electrodes: a theoretical study. J. Electroanal. Chem. 720-721, 92-100.

Bartlett, P.N., Birkina, P.R., Ghanema, M.A., 2000. Electrochemical deposition of macroporous platinum, palladium and cobalt films using polystyrene latex sphere templates. Chem. Commun. 17, 1671-1672.

Blodgett, K.B., 1935. Films built by depositing successive monomolecular layers on a solid surface. J. Am. Chem. Soc. 57, 1007-1022.

Butler, J.A.V., 1924. Studies in heterogeneous equilibria. part ii. The kinetic interpretation of the nernst theory of electromotive force. Trans. Faraday Soc. 19, 729-733.

Butler, J.A.V., 1932. The mechanism of overvoltage and its relation to the combination of hydrogen atoms at metal electrodes. Trans. Faraday Soc. 28, 379-382.

Do, T.Q.N., Varničić, M., Hanke-Rauschenbach, R., Vidaković-Koch, T., Sundmacher, K., 2014. Mathematical modeling of a porous enzymatic electrode with direct electron transfer mechanism. Electrochim. Acta 137, 616-626.

Ender, M., 2015. An extended homogenized porous electrode model for lithium-ion cell electrodes. J. Power Sources 282, 572-580.

Faraday, M., 1833. Experimental researches in electricity: third series. Philos. Trans. Royal Soc. Lond. 123, 23-54.

Ferguson, T.R., Bazant, M.Z., 2012. Nonequilibrium thermodynamics of porous electrodes. J. Electrochem. Soc. 159, 1967-1985.

Fick, A., 1995. On liquid diffusion. J. Membrane Sci. 100, 33-38.

Gray, W.G., 1975. A derivation of the equations for multi-phase transport. Chem. Eng. Sci. 30, 229-233.

Heim, M., Reculusa, S., Ravaine, S., Kuhn, A., 2012. Engineering of complex macroporous materials through controlled electrodeposition in colloidal superstructures. Adv. Funct. Mater. 22 (3), 538-545.

Jirkovský, J.S., Halasa, M., Schiffrin, D.J., 2010. Kinetics of electrocatalytic reduction of oxygen and hydrogen peroxide on dispersed gold nanoparticles. Phys. Chem. Chem. Phys. 12, 8042-8052.

Karajić, A., Reculusa, S., Heim, M., Garrigue, P., Ravaine, S., Mano, N., Kuhn, A., 2015. Bottom-up generation of miniaturized coaxial double electrodes with tunable porosity. Adv. Mater. Interfaces 2 (12).

Molina, A., Gonzalez, J., Laborda, E., Compton, R.G., 2013. On the meaning of the diffusion layer thickness for slow electrode reactions. Phys. Chem. Chem. Phys. $15,2381-2388$.

Reculusa, S., Ravaine, S., 2003. Synthesis of colloidal crystals of controllable thickness through the Langmuir-Blodgett technique. Chem. Mater. 15, 598-605. 
Reculusa, S., Heim, M., Gao, F., Mano, N., Ravaine, S., Kuhn, A., 2011. Design of catalytically active cylindrical and macroporous gold microelectrodes. Adv. Funct. Mater. 21 (4), 691-698.

Renslow, R., Donovan, C., Shim, M., Babauta, J., Nannapaneni, S., Schenk, J., Beyenal, H., 2011. Oxygen reduction kinetics on graphite cathodes in sediment microbia fuel cells. Phys. Chem. Chem. Phys. 13, 21573-21584.

Shinagawa, T., Garcia-Esparza, A.T., Takanabe, K., 2015. Insight on tafel slopes from a microkinetic analysis of aqueous electrocatalysis for energy conversion. Sci. Rep. 5 (13801), 1-21.

Szamocki, R., Reculusa, S., Ravaine, S., Bartlett, P.N., Kuhn, A., Hempelmann, R., 2006 Tailored mesostructuring and biofunctionalization of gold for increased electroactivity. Angew. Chem. Int. Ed. 45 (8), 1317-1321.

Tafel, J., 1905. Über die Polarisation bei kathodischer Wasserstoffentwicklung (concerning the polarisation during cathodic evolution of hydrogen). Zeit. Physik. Chem. 50A, 641-712.

Truesdell, C., Toupin, R., 1960. The Classical Field Theories. Springer-Verlag, New-York.
Valdés-Parada, F.J., Goyeau, B., Ochoa-Tapia, J.A., 2006. Diffusive mass transfer between a microporous medium and an homogeneous fluid: Jump boundary conditions. Chem. Eng. Sci. 61, 1692-1704.

Valdés-Parada, F., Aguilar-Madera, C., Álvarez Ramírez, J., 2011. On diffusion, dispersion and reaction in porous media. Chem. Eng. Sci. 66, 2177-2190.

Vidts, P.D., White, R.E., 1997. Governing equations for transport in porous electrodes. J. Electrochem. Soc. 144, 1343-1352.

Walcarius, A., Kuhn, A., 2008. Ordered porous thin films in electrochemical analysis. Trends Anal. Chem. 27, 593-603.

Wang, Y., Laborda, E., Ward, K.R., Tschulik, K., Compton, R.G., 2013. A kinetic study of oxygen reduction reaction and characterization on electrodeposited gold nanoparticles of diameter between $17 \mathrm{~nm}$ and $40 \mathrm{~nm}$ in $0.5 \mathrm{~m}$ sulfuric acid. Nanoscale 5, 9699-9708.

Whitaker, S., 1999. The Method of Volume Averaging. Kluwer Academic Publishers, Dordrecht, The Netherlands.

Zhang, J., 2008. PEM Fuel Cell Electrocatalysts and Catalyst Layers, chapter: Electrocatalytic Oxygen Reduction Reaction. Springer, London. 